\title{
REMISE EN QUESTION DE L'ÂGE DE LA CAPTURE DE LA MEUSE LORRAINE À LA LUMIÈRE DE LA RÉPARTITION DES AMPHIBOLES DES VOSGES DANS LES TERRASSES MOSANES
}

\author{
Étienne JUVIGNÉ, Geoffrey HOUBRECHTS
}

\begin{abstract}
Résumé
Les associations de minéraux denses transparents de 25 lambeaux de divers niveaux de terrasses mosanes ont été établies et les résultats antérieurs en cette matière ont été rassemblés. Les amphiboles présentes dans la classe granulométrique de 75 à $425 \mu \mathrm{m}$ des terrasses de cailloutis mosans proviennent des Vosges, qu'elles soient brunes ou vertes assorties de pléochroïsme. De la sorte, il apparait qu'au cours de l'incision de la Meuse, les amphiboles des Vosges sont entrées en scène dans une terrasse située à $128 \mathrm{~m}$ à Givet (+28 m vs plaine d'inondation) et à 134-140 m à Lixhe/Visé (+80 m vs plaine d'inondation). Cette terrasse, par ailleurs en contre-pente, débouche dans le profil de référence de Maastricht au niveau de la Terrasse de Valkenburg-1 pour laquelle il existe une estimation d'âge de 1,57 Ma. La capture de la Meuse lorraine aurait donc eu lieu dans la seconde moitié du Pléistocène inférieur et non pendant la transition mio-pliocène comme avancé dans la littérature la plus récente.
\end{abstract}

Mots-clefs

Belgique, Meuse, Vosges, Moselle, terrasses, capture, amphibole, Quaternaire

\begin{abstract}
The associations of transparent heavy minerals of 25 shreds of the various levels of terraces have been established and the previous results in this matter have been collected. The amphiboles present in the grain size from 75 to $425 \mu \mathrm{m}$ of the alluvia of the Meuse terraces originate from the Vosges, whether they are brown or green including pleochroism. In this way, it appears that during the incision of the Meuse, the amphiboles of the Vosges entered the scene in a terrace located at $128 \mathrm{~m}$ in elevation at Givet ( $+28 \mathrm{~m}$ above flood plain) and at 134-140 m at Lixhe/Visé (80 $\mathrm{m}$ above flood plain). This terrace, by the way in reverse slope, leads into the Maastricht reference profile at the Valkenburg-1 Terrace, for which there is an estimated age of $1.57 \mathrm{Ma}$. The capture of the Lorraine Meuse would therefore have occurred in the second half of the lower Pleistocene and not during the mio-pliocene transition as the most recent literature says.
\end{abstract}

Keywords

Belgique, Meuse, Vosges, Moselle, terrasse, capture, amphibole, Quaternary

\section{INTRODUCTION}

Remarque préliminaire. Les sites évoqués avant l'exposé des résultats sont mis en évidence sur la Figure 1.

Quels que soient les mécanismes proposés par les différents auteurs pour expliquer la traversée du Massif de Rocroi par la Meuse, il y a unanimité dans la littérature pour placer cet événement avant le Quaternaire (Figure 1). Rutot (1897) invoque un affaissement de l'Ardenne inhérent à la transgression miocène pour faire passer la Meuse par une pente naturelle via Revin pour rejoindre Givet et poursuivre ainsi son cours suivant son itinéraire actuel. Dollfus (1900) attribue la mise en place de ce tronçon à une capture provoquée par la «Meuse de Fumay qui est venue soutirer la Meuse de Mézières ». Pissart (1961) se range parmi les auteurs qui défendent le processus de capture, et il placera celle de la Meuse lorraine pendant la transition mio-pliocène (Pissart, 1974 ; Pissart et al., 1997). On notera que cette hypothèse implique que la capture de la Semois par la Meuse de Dinant soit plus ancienne, mais l'auteur ne se prononce pas sur l'âge du détournement de ce cours d'eau.

Juvigné et al. (2020a, 2020b) étudiant les très hautes terrasses mosanes depuis Namur jusqu'à Huy concluent que la capture de la Semois devrait être enregistrée dans le lambeau de terrasse (lbt) de Paradis/Couthuin (Figure 1) dont la base est à 


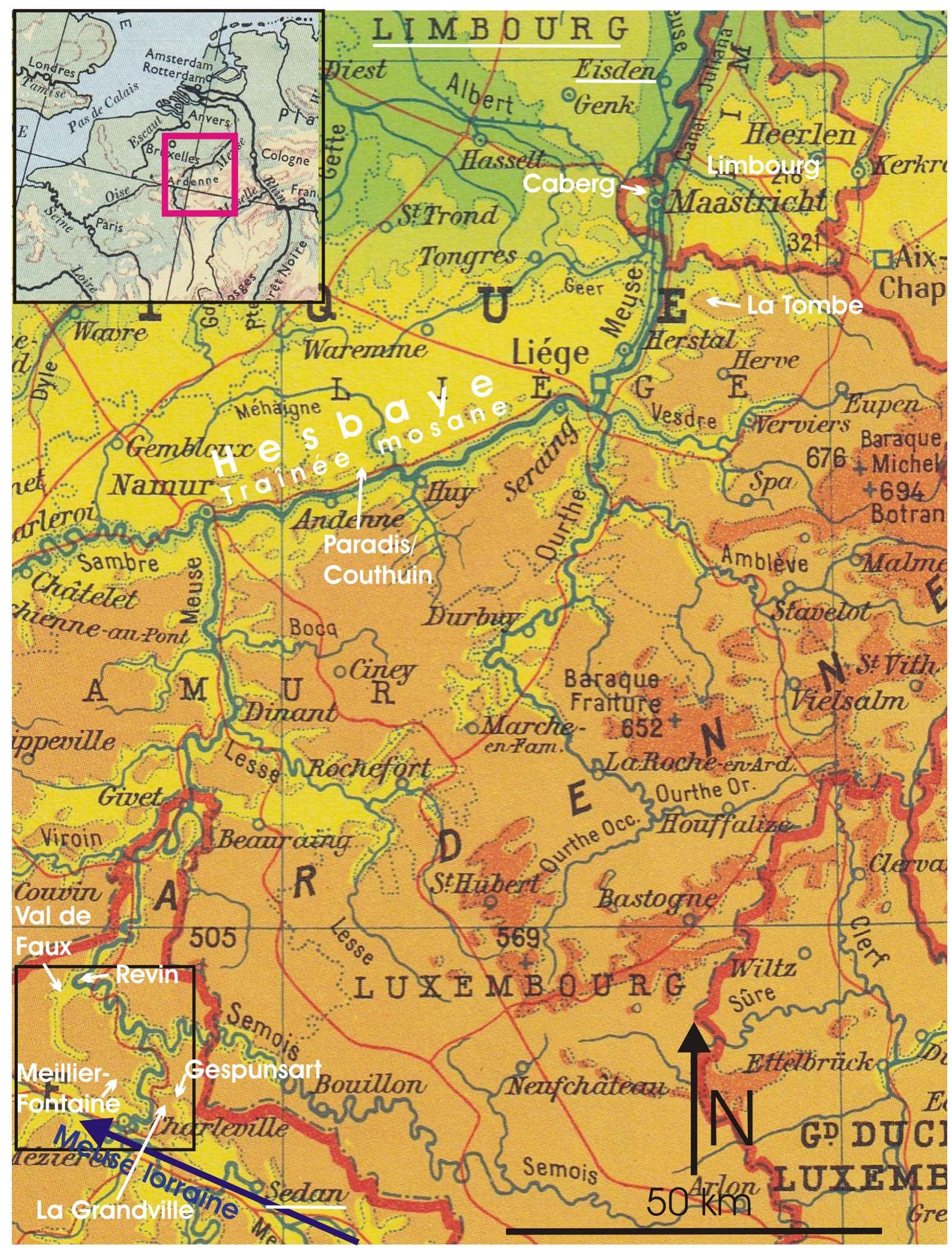

Figure 1. Localisation de sites évoqués dans le présent travail. (A) Le cours de la Meuse depuis Sedan (France) jusqu'au Limbourg (Belgique et Pays-Bas) (Fond de carte de l'Atlas Mantnieks, 1955). Explications : (1) la représentation de la Meuse lorraine est caricaturale, elle représente l'hypothèse selon laquelle à partir de la région de Charleville, le cours d'eau primitif poursuivait peut-être son cours vers le NO par la vallée de la Sormonne ; (2) le cadre noir délimite la région des captures évoquées dans la littérature entre Sedan et Revin. Elle est présentée en détails à la Figure 9 ; (3) en blanc, mise évidence de localités et position de la Traînée mosane. 
des amphiboles des Vosges dans les terrasses mosanes

$175 \mathrm{~m}$, le sommet à $179 \mathrm{~m}$, et la dénivelée par rapport à la plaine d'inondation de $105 \mathrm{~m}$ (en abrégé : Cou-Par[b175; s179; d105]). Ce lbt est aussi 40 m plus bas que la terrasse la plus élevée dite Traînée mosane par Macar (1945a).

Le présent travail va montrer que les amphiboles des Vosges entrent en scène dans une terrasse plus basse que celle de Paradis/Couthuin, si bien qu'il existe donc une succession lithostratigraphique cohérente pour défendre l'idée que les captures de la Semois puis de la Meuse lorraine ont eu lieu pendant le Quaternaire plutôt que pendant la transition mio-pliocène.

\section{SÉMANTIQUE ET ABRÉVIATIONS}

Le chapitre équivalent dans Juvigné et al. (2020b) est d'application dans le présent travail. Deux mises au point particulières sont à ajouter.

Meuse lorraine. L'expression Meuse lorraine a été couramment utilisée dans la littérature depuis Pissart (1961) jusqu'à Juvigné et al. (2020a) pour désigner le cours d'eau qui, selon Pissart (1961; 1997a) a été capturé à Nouzonville à 300 m d'altitude par la Meuse de Dinant. En réalité, à ce stade, la Meuse n'était qu'un petit affluent de la Haute Moselle laquelle recevait entre Sedan et Charleville, la Bar qui n'avait pas encore été amputée de la partie supérieure de son bassin (de Davis, 1895 à Harmand, 1992). La dénomination précitée est donc largement abusive, mais elle a le mérite de faire référence aux travaux discutés plus bas.

Hornblende et amphibole. Dans la littérature antérieure relative aux terrasses mosanes, le terme hornblende a été utilisé abusivement pour désigner une amphibole présente dans les dépôts mosans, mais elle n'a jamais été déterminée comme telle par les auteurs qui en ont fait usage. En nous référant à la classification formelle des amphiboles (Leake, 1997), nous utiliserons 'amphibole', mais nous respecterons l'usage dans les évocations d'auteurs.

\section{REVUE DE LA LITTÉRATURE EN RE- LATION AVEC LE SUJET TRAITÉ}

En étudiant les minéraux des sables des terrasses de la Meuse du Limbourg néerlandais, Zonneveld (1949) identifie des hornblendes brun-vert en pré- cisant qu'il s'agit d'une découverte, mais il ne peut se prononcer sur l'origine géographique du minéral.

En étendant l'étude précitée aux terrasses de la Meuse en Belgique, Bustamante (1973) attribue une origine vosgienne à cette hornblende. L'entrée en scène de celle-ci a lieu dans la $\mathrm{T}$. de La Tombe (à Dalhem sur le flanc oriental de la Basse-Meuse liégeoise : Dal-LaT[b129 ; s139 ; d73). Dans son modèle, cette terrasse est la troisième à partir de la Traînée mosane dans l'ordre chronologique. L'auteur admettant l'âge mio-pliocène de la capture de la Meuse lorraine et de sa propre terrasse la plus élevée, considère que l'absence du minéral dans les deux terrasses supérieures est due à l'altération qu'il a subie dans les environnements chauds et humides du Tertiaire. Par ailleurs, l'auteur établit une relation entre la capture de la Moselle survenue à Toul et la chute de fréquence du minéral au niveau d'une basse terrasse mosane du Limbourg belge, la T. d'Eisden-Lanklaar placée in illo tempore dans le Riss II par Paulissen (1973). Bustamante (1976, 1986) n'apportera pas de modification à l'état des connaissances en matière de hornblende dans les dépôts mosans.

Bustamante et Voisin (1975) trouvent des hornblendes des Vosges dans le cailloutis supérieur de la carrière de La Grandville (à 273 m), mais ils ne trouvent que des matériaux d'origine ardennaise dans le cailloutis du col de Meillier-Fontaine (NDR : à 310-315 m) et dans un lbt du Val de Faux $60 \mathrm{~m}$ plus haut que le fond de vallée. Dès lors, ils invitent à revoir l'évolution du paléo-réseau hydrographique défendu par Pissart $(1961,1974)$ dans la zone des captures.

Krook (1993), étudie en détail les minéraux des sables dans une séquence de la T. de Caberg/ Maastricht qui précède immédiatement celle $\mathrm{T}$. d'Eisden-Lanklaar dans la stratigraphie. Il constate que la chute de fréquence de la hornblende des Vosges a lieu au sein de la T. de Caberg.

Bustamante (1995) précise que c'est un «petit cours d'eau » qui est responsable de la mise en place des dépôts fluviatiles de type ardennais à Meillier-Fontaine et dans le Val de Faux (cf. supra).

Krook (in Pissart et al., 1997a) produit des diagrammes de l'évolution de la fréquence des minéraux denses transparents dans une séquence 
de terrasses de la région de Givet, distribuées sur une dénivelée de $100 \mathrm{~m}$ par rapport à la plaine d'inondation. Les minéraux sont répartis en deux classes granulométriques de 32 à $53 \mu \mathrm{m}$ et de 53 à $420 \mu \mathrm{m}$. Dans 1'ensemble, la fréquence de la hornblende des Vosges est élevée dans les terrasses comprises entre $+28 \mathrm{~m}$ et $+6 \mathrm{~m} /$ plaine d'inondation. Les auteurs acceptent que les hornblendes ont disparu par altération dans les terrasses au-dessus de $+28 \mathrm{~m}$, et que la chute de fréquence sous la terrasse de $+6 \mathrm{~m}$ est la conséquence de la capture de la Moselle à Toul.

\section{MÉTHODES}

Les éléments utilisés antérieurement pour identifier l'origine vosgienne d'une partie des alluvions mosanes en Belgique, peuvent être des cailloux de granite, des hornblendes brunes et/ou des zircons zonés (Bustamante, 1973). Cet auteur ne cite que très occasionnellement la présence de cailloux roulés de granite dans les sites où il fait essentiellement état de minéraux vosgiens. Nous avons nousmêmes constaté que la fréquence des cailloux de granite est réduite à l'état de traces $(<1 \%)$ dans les terrasses mosanes revisitées. Nous nous limiterons donc comme les autres auteurs qui ont traité précédemment le sujet, à la détermination des minéraux denses transparents.

Pour notre part, nous n'avons pas retenu le zircon zoné comme élément représentatif de l'origine vosgienne. En effet, nous ne contestons pas la pertinence de son utilisation dans les alluvions fluviatiles à l'aval immédiat des Vosges ou à la traversée des terrains du Bassin Parisien, mais la zonation d'un cristal correspond à des figures de croissance, elle est donc nécessairement azonale. Par contre, en extrayant les minéraux denses d'un broyat d'une quinzaine de cailloux roulés de granites différents (du lit de la Moselle à Metz); les amphiboles extraites étaient vert clair à vert plus foncé ou vertes à vert brunâtre ; toutefois, les variations de couleur dans le microscope polarisant existent pour chaque amphibole dépendant de la position du minéral dans le frottis (pléochroïsme). On notera que Beiner et al. (2009) distinguent dans le Massif vosgien trois catégories d'amphiboles : des amphiboles vertes ou brunes avec une extinction de 12 à $34^{\circ}$, une amphibole brun vert à jaune vert avec un angle d'extinction faible (5 à $\left.12^{\circ}\right)$ et une amphibole basaltique brun foncé avec un angle d'extinction très faible. Ceci justifie également la prise en considération des amphiboles vertes pour la recherche de la liaison des terrasses mosanes avec les Vosges, mais la mesure de l'angle d'extinction s.s. fait appel à une technique inapplicable dans les déterminations en série.

Pour utiliser également les amphiboles vertes comme minéral-guide, il y a lieu d'éliminer celles appartenant aux autres sédiments qui préexistent dans le bassin de la Meuse. Les travaux de Bourguignon (1954) sur l'ensemble des sables des Hautes Fagnes (altérites du socle paléozoïque, Crétacé, Oligocène), et ceux de Demoulin (1987) sur les sables oligocènes des Hautes Fagnes et de la région liégeoise, montrent que les amphiboles ne se présentent que très sporadiquement dans l'ensemble des terrains meubles précités, et que leur fréquence n'atteint jamais $1 \%$. Tavernier (1947) mentionne la présence sporadique de hornblendes dans des terrains marins qui vont de l'Éocène jusqu'au Pliocène, mais il s'agit toujours de sites en Flandre et la granularité dans laquelle les déterminations ont été réalisées n'est pas précisée.

Par contre, dans les limons lœssiques notamment weichseliens et saaliens, la fréquence des amphiboles vertes dans les spectres de minéraux denses transparents s'expriment jusqu' en dizaines de pourcent (Pirson et al., 2017), et ce matériau se retrouve nécessairement dans la matrice des dépôts fluviatiles. Un test a été réalisé sur du lœss weichselien dont le spectre de minéraux denses transparents contient jusqu'à $40 \%$ d'amphiboles vertes (Juvigné, 1978), il en ressort que les amphiboles vertes du lœss sont toutes éliminées par un tamisage à $75 \mu \mathrm{m}$. Toutefois, il faut aussi veiller à éviter le prélèvement d'échantillons pouvant contenir les amphiboles brun clair à brun foncé (kaersutite, pargasite) bien connues dans les retombées volcaniques weichseliennes des téphras du Laacher See et de Rocourt (ex. Juvigné, 1993). Ceci implique que l'on évite les prélèvements de surface dans des cailloutis dont le toit est en affleurement dans les zones de retombées ou que l'on en tienne compte au cours des déterminations. Cette dernière réserve est particulièrement pertinente en cas de prélèvement dans les très basses terrasses qui contiennent de tels minéraux dans leurs cailloutis (Juvigné, 1979).

Dans ces conditions, on peut accepter que les amphiboles de plus de $75 \mu \mathrm{m}$ vertes ou brunes présentes 
des amphiboles des Vosges dans les terrasses mosanes

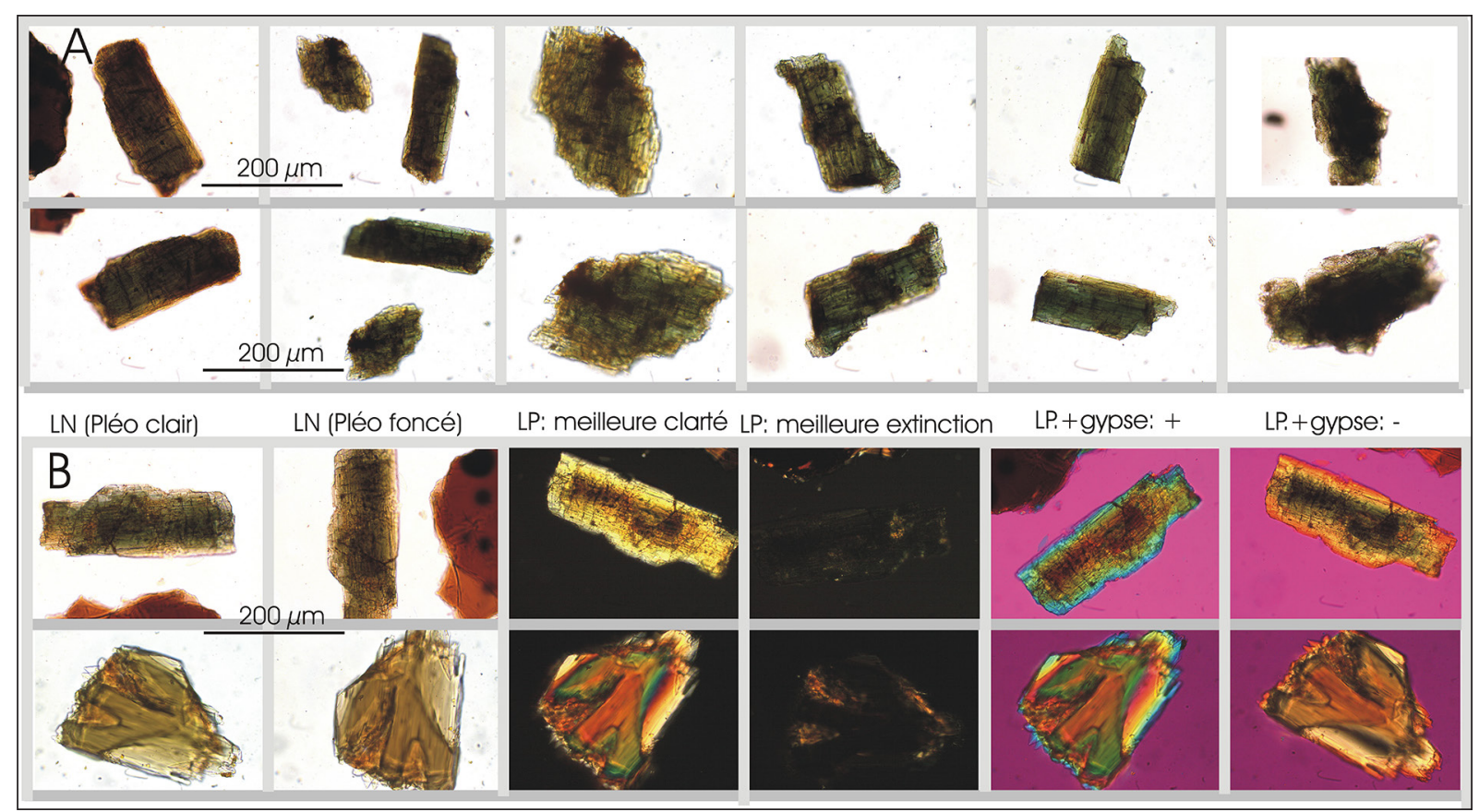

Figure 2. Amphiboles en frottis dans le microscope polarisant. (A) Extraites d'un broyat d'éclats d'une quinzaine de cailloux roulés de granites provenant du lit de la Moselle à Metz. (B) Extraites du lbt de Chemin d'Erpent à Namur-Est : Nam-ChE[b110; s115 m; d30]. On notera que les couleurs du retard du minéral de la rangée inférieure attestent une épaisseur nettement supérieure à celle des autres amphiboles présentées.

dans les sables mosans ne peuvent provenir que des Vosges. En conséquence, tous les échantillons ont été traités comme suit : dispersion dans $\mathrm{HCl}_{10 \% \text { vol }}$; rinçage ; tamisage sous courant d'eau à $425 / 75 \mu \mathrm{m}$; séchage ; séparation d'une aliquote de $\sim 10 \mathrm{~g}$ dans le bromoforme en ampoule, en répétant les cycles 'agitation- décantation- récupération' jusqu'à épuisement du surnageant ; montage des frottis ; détermination suivant la méthode du ruban sous couvre-objet strié ; détermination avec répartition en trois classes granulométriques : 75-150 $\mu \mathrm{m}$; 150-300 $\mu \mathrm{m} ; 300-425 \mu \mathrm{m}$; calcul des fréquences sur l'ensemble des trois classes granulométriques, car la part de minéraux denses est très faible, voire nulle, dans le classe de 300 à $425 \mu \mathrm{m}$, et trop faible pour calculer des pourcentages fiables dans la classe 150-300 $\mu \mathrm{m}$.

Les résultats publiés par Bustamante (1973) sont présentés par classe granulométrique suivant un pas de demi-phi de phi4,5 à phi 2 ; ils ont donc pu être recalculés pour être comparables aux nôtres (de phi3 à phi2). Par contre, les résultats de Krook (op. cit.) sont présentés d'une part pour la fraction limoneuse (32 à $53 \mu \mathrm{m}$ ) et d'autre part pour la fraction sableuse (53-420 $\mu \mathrm{m})$ et ceux de Zonneveld (1949) ne sont disponibles qu'en graphique de fréquence sans précision relative à la taille des grains déterminés. Dans les deux derniers cas, les comparaisons n'ont qu'une valeur semi-quantitative et des amphiboles des lœss sont nécessairement présentes. L'ensemble des spectres de minéraux denses transparents calculables sont collationnés dans le Tableau 1 (annexe).

\section{RÉSULTATS}

Dans le présent chapitre, nous allons passer en revue la répartition stratigraphique des amphiboles dans les terrasses mosanes en commençant par nos résultats personnels obtenus dans la région de Namur jusqu'à Huy et en Basse-Meuse liégeoise. Les données produites par les autres déterminateurs précités seront alors discutées.

\section{A. Le tronçon de Namur jusqu’à Huy}

Il a été rappelé plus haut que c'est dans ce tronçon que Juvigné et al. (2020a\&b) ont estimé avoir identifié l'entrée en scène des alluvions de la Semois dans la T. de Paradis/Couthuin (CouPar[b175; s179; d105]) tout en ayant constaté l'absence de matériaux vosgiens dans cette même terrasse ( 7 échantillons et $n>1000$ ) et dans toutes les autres plus élevées. Dès lors, il s'imposait de rechercher les amphiboles dans les terrasses plus basses (Figure 3). Aucune amphibole n'a été trouvée dans les échantillons des lbxt étagés 


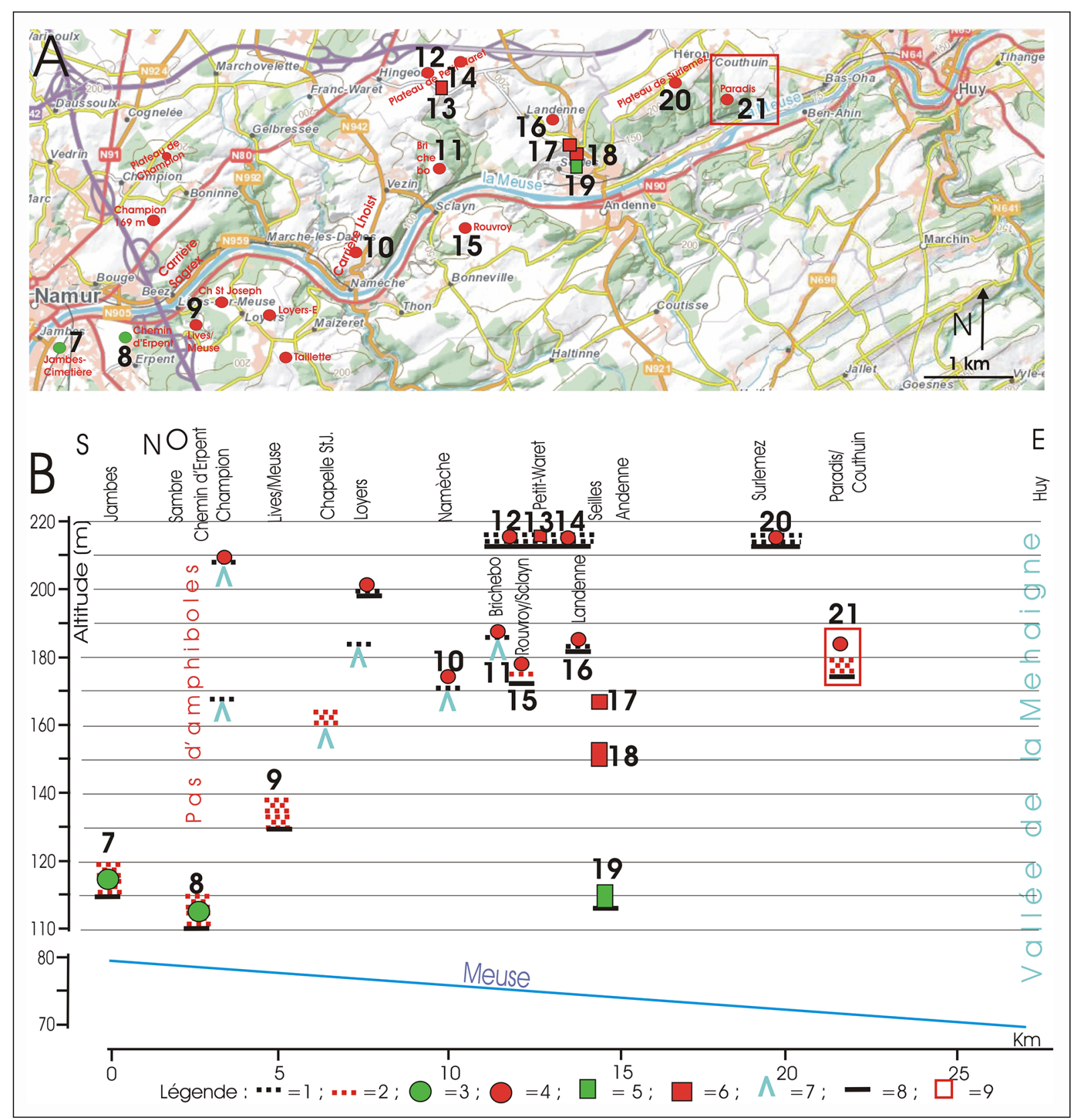

Figure 3. (A) Localisation géographique des lbxt étudiés entre Namur et Huy. (B) Position altimétrique des lbxt revisités. Légende : 1= cailloutis de type $O n x ; 2=$ cailloutis de type $q 2 m ; 3=$ présence d'amphiboles dans le cailloutis (détermination personnelle : EJ) ; 4= absence d'amphiboles dans le cailloutis (détermination personnelle : EJ) ; 5= présence d'amphiboles dans le cailloutis (détermination Bustamante, 1973) ; 6= absence d'amphiboles dans le cailloutis (détermination Bustamante, 1973). 7= socle de roches solubles (calcaire et/ou dolomie) ; $8=$ socle de roches siliceuses et/ou argileuses ; 9= lbt de référence de Paradis/Couthuin. Les numéros des sites correspondent à ceux du Tableau 1 (annexe). N.B. La description de tous les sites de prélèvement est disponible dans Juvigné et al. (2020b).

en gradins depuis les cailloutis $O n x$ de plateau (exemple Surlemez/Couthuin : Cou-Sur[b213 ; s217 ; d141]) jusqu'au lbt de Lives-sur-Meuse (b135; s140 ; d56). Par contre, les amphiboles sont présentes dans le spectre de minéraux denses transparents des échantillons : (1) du cimetière de Jambes (Namur sud : Jam-Cim[b115; s120 ; d39] ; profondeur de l'échantillon $200 \mathrm{~cm}$, soit à $118 \mathrm{~m}): 8,8 \%$ d'amphiboles vert brunâtre et $1,2 \%$ de vertes [ $\mathrm{n}=167])$; (2) de Chemin d'Erpent (Namur-Est : Nam-ChE[b110 ; s115 m ; d31], échantillon prélevé au contact du socle à $110 \mathrm{~m}$ ) : $4,5 \%$ d'amphiboles vert brunâtre et $4 \%$ de vertes [ $\mathrm{n}=190])$; (3) dans la carrière Lhoist de Seilles (Sei-Lho[b113; s117; d38], 8,3\% de hornblendes des Vosges). 
des amphiboles des Vosges dans les terrasses mosanes

\section{B. Dans la Basse-Meuse liégeoise}

Pendant la durée de ce travail, des affleurements de cailloutis ont été accessibles dans des localités de la Basse-Meuse liégeoise (Figure 4); des échantillons y ont été prélevés et des frottis de minéraux denses ont été préparés. Les résultats des déterminations sont rassemblés dans le Tableau 1 (annexe). Les valeurs de sites étudiés par Bustamante (1973) ont pu être adaptées et intégrées (cf. supra).

Sur le flanc gauche de la vallée, il n'existe aucun lbt plus élevé que celui de Lixhe (b134 ; s140 ; d78 m), et tous contiennent des amphiboles. Sur le flanc droit, les amphiboles entrent en scène dans la terrasse de La Tombe/Dalhem (b129; s139; d73) et leur présence est continue dans les terrasses plus basses, tandis que tous les lambeaux plus élevés sont dépourvus du minéral. Une réserve doit être émise pour les trois niveaux de Bois de Breux dont les cailloutis pourraient relever uniquement de l'Ourthe, et n'auraient donc aucune raison de contenir des amphiboles (Juvigné et Van Campenhout, 2020).

\section{Synthèse}

À Namur, la présence des amphiboles est avérée dès la terrasse correspondant au lbt du cimetière de Jambes où le toit du cailloutis $q 2 \mathrm{~m}$ est à $120 \mathrm{~m}$ d'altitude, soit $40 \mathrm{~m}$ plus haut que la plaine d'inondation. Par contre, aucune amphibole n'a été trouvée dans les cailloutis plus haut que la base de la terrasse du lbt de Lives-sur-Meuse, c'est-à-dire à partir de $130 \mathrm{~m}$ d'altitude, soit $51 \mathrm{~m}$ plus haut que la plaine alluviale actuelle. La limite entre les terrasses à amphiboles et celles qui en sont dépourvues se trouve donc entre 39 et $51 \mathrm{~m}$ plus haut que la plaine d'inondation entre Namur et Huy. Dans la BasseMeuse liégeoise, le lbt le plus élevé contenant les

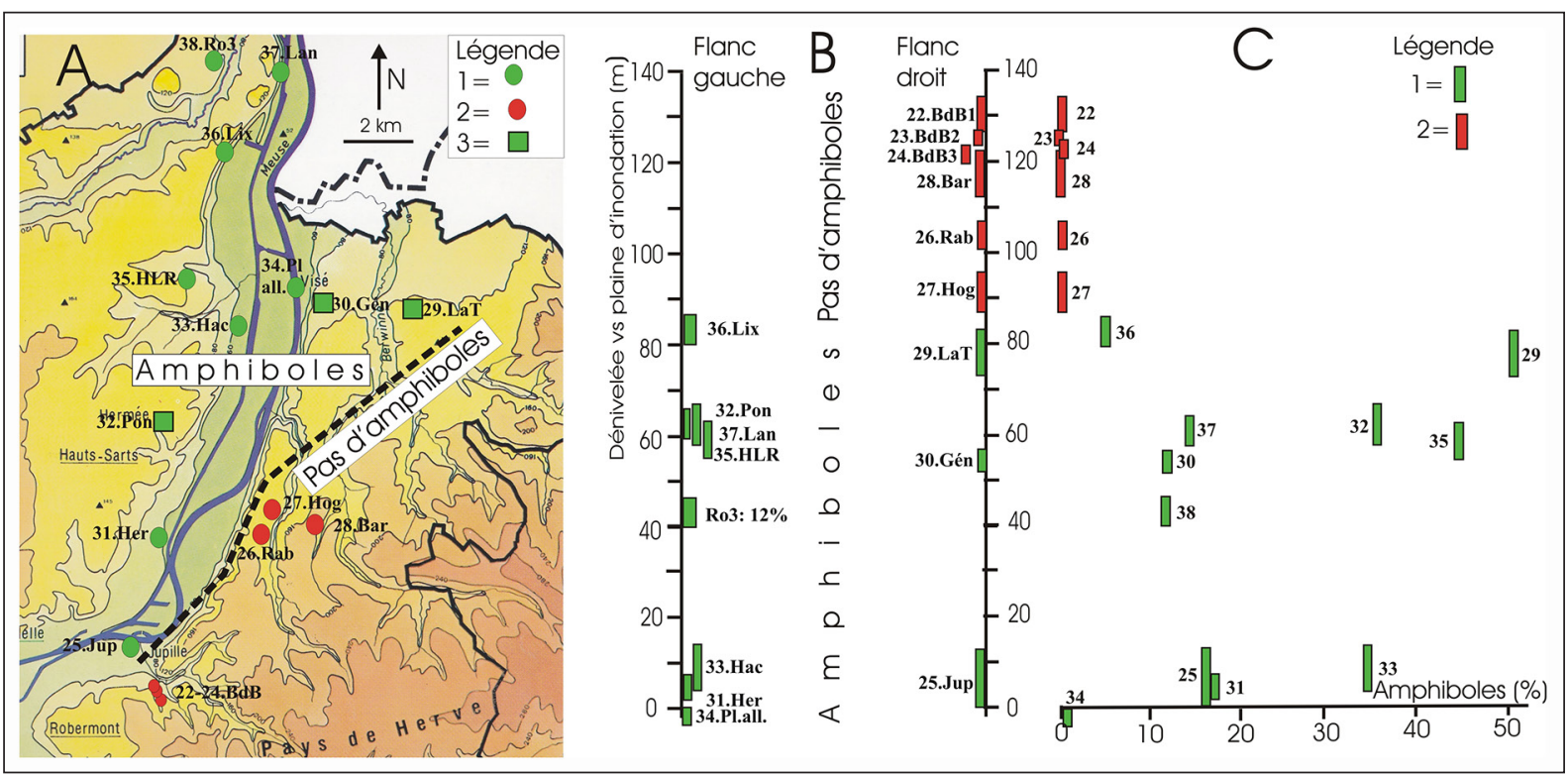

Figure 4. Les amphiboles des Vosges dans des terrasses de la Basse-Meuse liégeoise (les numéros des sites correspondent à ceux du Tableau 1 en annexe). (A) Localisation des sites étudiés, en rouge sans amphiboles et en vert avec amphiboles : (1) flanc gauche du sud au nord : Her= Herstal, Hall Omnisport (b62 ; s67 ; d2) ; Pon= Pontisse, ancienne gravière (b118; s124; d60; détermination Bustamante, 1973); Hac $=$ Haccourt, versant $(\mathrm{b} 60 ; \mathrm{s} 72 ; \mathrm{d} 3)$; $\mathrm{HLR}=$ Heure-le-Romain, carrière de craie (b112; s120; d55); Pl. allu= plaine alluviale à Hermalle ( $\mathrm{s} 56$; prélèvement à $53 \mathrm{~m})$; Lix = Lixhe, carrière de craie (b134; s140; d78); Lan= Lanaye, carrière de marne (b110; s118; d58); Ro3= carrière du Romont/CBR en 2017 (b92 ; s98; d40). (2) flanc droit du sud au nord : BdB1= Bois de Breux 1 (b186; s193; d126); BdB2= Bois de Breux 2 (b183; s186; d123); BdB3= Bois de Breux 3 (b178; s183; d118); Jup= Jupille, tranchée d'impétrants (b60;s72; d0) ; Rab= Rabosée, cimetière (b159; s164; d101) ; Bar= Barchon, Sortie E40 (b170 ; s180 ; d113) ; Hog= Hognée, tranchée E40 (b145 ; s154 ; d86). Trois sites étudiés par Bustamante (1973) sont intégrés après adaptation des fréquences (cf. supra) : Pontisse (b118; s124; d60) ; LaT= La Tombe, ancienne gravière $(\mathrm{b} 129 ; \mathrm{s} 139 ; \mathrm{d} 73)$; Gén= Génistreux, ancienne gravière (b108; s112; d52). Légende : 1= lbt avec amphiboles (E. Juvigné) ; 2= lbt sans amphiboles (E.J.) ; 3=lbt avec amphiboles (L. Bustamante) ; (B) Altitude relative des sites par rapport à la plaine d'inondation ; les colonnes verticales représentent l'épaisseur des cailloutis mosans en vert avec amphiboles, en rouge sans amphiboles. Légende : $1=$ sans amphiboles ; $2=$ avec amphiboles ; (C) Fréquence des amphiboles dans les sites étudiés ( $c f$. Tableau 1 en annexe). 
premières amphiboles est celui de la carrière de craie CBR de Lixhe : Lix-CBR[134 ; s140; d78 m]. Dans l'état actuel des connaissances, le profil longitudinal de la terrasse dans laquelle les amphiboles des Vosges entre en scène serait donc en légère contre-pente entre Namur et Lixhe/Visé.

\section{DISCUSSION}

\section{A. Intégration critique de sites décrits antérieu- rement}

Des résultats publiés par Krook (in Pissart et al., 1997a\&b), Bustamante (1973) et Zonneveld (1949) sont revisités ci-dessous.

\section{Les hornblendes dans des terrasses de la région de Givet}

Krook (in Pissart et al., 1997a : Figure 1, p.412) a publié un diagramme de référence de la distribution stratigraphique des minéraux denses transparents dans les terrasses de la région de Givet depuis le niveau de la Meuse (altitude de la plaine d'inondation : $100 \mathrm{~m}$ ) jusqu'à la plus haute terrasse dont la base est à $200 \mathrm{~m}$ (100 m plus haut). Ce diagramme montre que les hornblendes brunes des Vosges entrent en scène dans la terrasse située à +28 $\mathrm{m}$, elles atteignent des fréquences de 10 à $30 \%$ jusqu'à la terrasse de $+6 \mathrm{~m}$, puis elles disparaissent en-dessous. Les auteurs ont mis à juste titre cette disparition en relation avec la capture de la Moselle survenue à Toul, mais ils n'ont accordé aucun intérêt à la position de leur entrée en scène puisque dans le travail, il est acquis dès le départ que la Meuse lorraine traverse le Massif de Rocroi depuis la transition mio-pliocène. L'absence du minéral dans les hautes terrasses est ainsi justifiée depuis Bustamante (1973) par l'altération physico-chimique que les en a fait disparaître. Par ailleurs, dans ce travail les hornblendes vertes sont à juste titre distinguées des hornblendes brunes des Vosges dans la mesure où les déterminations ont été pratiquées à partir de $53 \mu \mathrm{m}$, si bien que des amphiboles vertes des lœss sont donc nécessairement présentes dans la partie de la matrice d'origine lœssique des lbxt (cf. supra).

\section{Les hornblendes dans des terrasses depuis Dinant jusqu'à Namur}

Bustamante (1973) a examiné la répartition stratigraphique des hornblendes brunes dans plusieurs lbxt entre Dinant et Namur (Figure 5).

Les hornblendes brunes sont présentes dans les lbxt dont la dénivelée par rapport à la plaine d'inondation est inférieure à $37 \mathrm{~m}: 4,4 \%$ à Profondville (b122; d37), 17,8 \% à Hun (b121; d34), 1,8 \% à Tailfer (b90; d5) ; elles sont absentes dans deux lbxt nettement plus élevés : Hun (b151, d64), Wépion (b200; d116). L'auteur explique la chute de

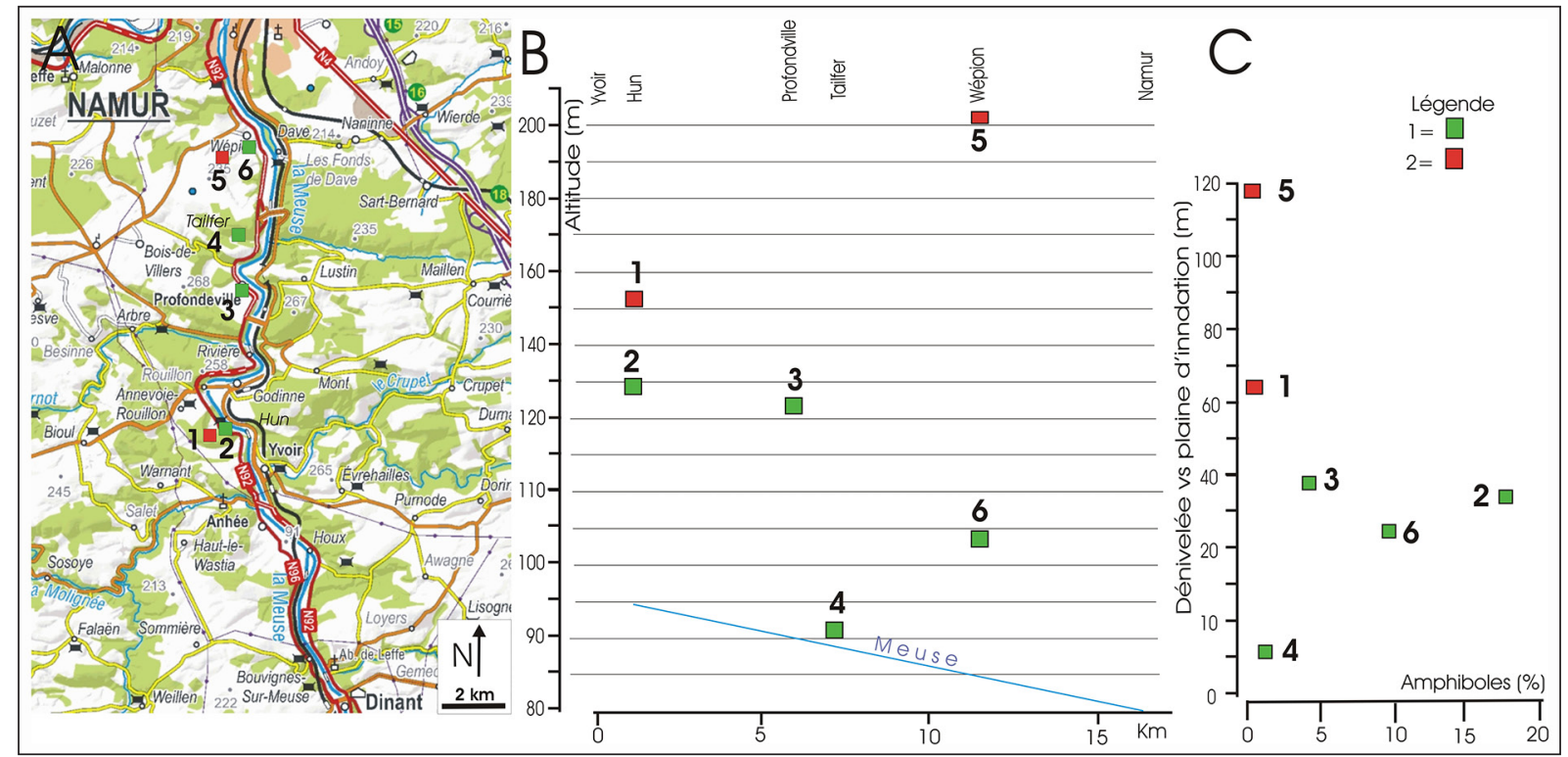

Figure 5. Répartition des hornblendes des Vosges dans des terrasses de la Meuse entre Dinant et Namur d'après Bustamante (1973). (A) Localisation des sites étudiés. (B) Position en altitude des sites de prélèvement. (C) Dénivelée des sites de prélèvement par rapport à la plaine d'inondation. Les numéros des sites correspondent à ceux du Tableau 1 (en annexe). Légende : 1= lbt avec amphiboles ; $2=1 b t$ sans amphiboles. 
des amphiboles des Vosges dans les terrasses mosanes

fréquence dans la très basse terrasse de Tailfer par sa postériorité par rapport à la capture de la Moselle à Toul. Par contre, il n'accorde aucune importance au niveau de leur entrée en scène, puisque dans l'ensemble de son travail il attribue à l'altérabilité du minéral son absence au-delà des moyennes et des basses terrasses. Bustamante (1995) en se préoccupant de la relation entre la fréquence des seules hornblendes brunes et la capture de la haute Moselle à Toul n'a même plus fait allusion à ses observations faites à Hun et Profondville.

\section{Les hornblendes dans les terrasses du Limbourg belge}

Dans le Limbourg belge (flanc gauche de la vallée de la Meuse), la terrasse mosane la plus élevée est le vaste Plateau de Campine dont le toit du cailloutis est entre 40 et $50 \mathrm{~m}$ plus haut que la plaine d'inondation entre Maastricht et Maaseik. Entre ce plateau et la plaine alluviale, il existe une série de terrasses en gradins (Paulissen, 1973) dans lesquelles la présence des hornblendes des Vosges est généralisée (Bustamante, 1973) (Figure 6). L'auteur attribue à la capture de la Haute Moselle, la diminution drastique de fréquence entre la T. de Caberg-Pietersem et celle d'Eisden-Lanklaar.

\section{Les amphiboles dans les terrasses du Limbourg néerlandais}

Zonneveld (1949) produit une étude de la répartition stratigraphique des minéraux denses transparents dans les terrasses mosanes du Limbourg néerlandais en s'alignant sur le modèle des terrasses de Brueren (1945) (Figure 7). C'est ainsi qu'il a le premier signalé la présence systématique de hornblendes brunes et vertes dans tous les niveaux de terrasse à partir de la $\mathrm{T}$. de Margraten (base à $152 \mathrm{~m} ; \mathrm{d}=107)$; toutefois l'auteur écrit ne pas pouvoir proposer une provenance pour ce minéral. C'est Bustamante (1973) qui, le premier, en a cité l'origine vosgienne (cf. supra). On notera qu'en terme d'altitude, le cailloutis de Margraten est nettement plus élevé que le plus haut niveau à amphibole de la Basse-Meuse liégeoise (rappel : Dal-LaT[b129; s139; d73). Qui plus est, Zonneveld mentionne également la présence de hornblendes brunes et vertes dans le lambeau de Simpelveld dont la base est connue à $\sim 160 \mathrm{~m}(\mathrm{~d}=\sim 115)$, ce qui soulève nécessairement la question d'une déformation du sol entre Margraten et Simpleveld. Enfin, on notera que Zonneveld précise que les hornblendes sont absentes dans les terrasses de Kosberg, Crapoel et Noorbeek-Eygelshoven entre la Basse-Meuse liégeoise et la Geul, toutes plus élevées que la T. Margraten (Ostmaas ; Hol, 1949).

\section{Synthèse : répartition stratigraphique des amphiboles depuis Givet jusqu'au Limbourg}

Tous les résultats relatifs à la fréquence des amphiboles sont rassemblés dans la Figure 8. Sur le dernier modèle en date des terrasses de la Meuse, la distinction est ajoutée entre les terrasses à amphiboles et celles qui en sont dépourvues. Dans la série des

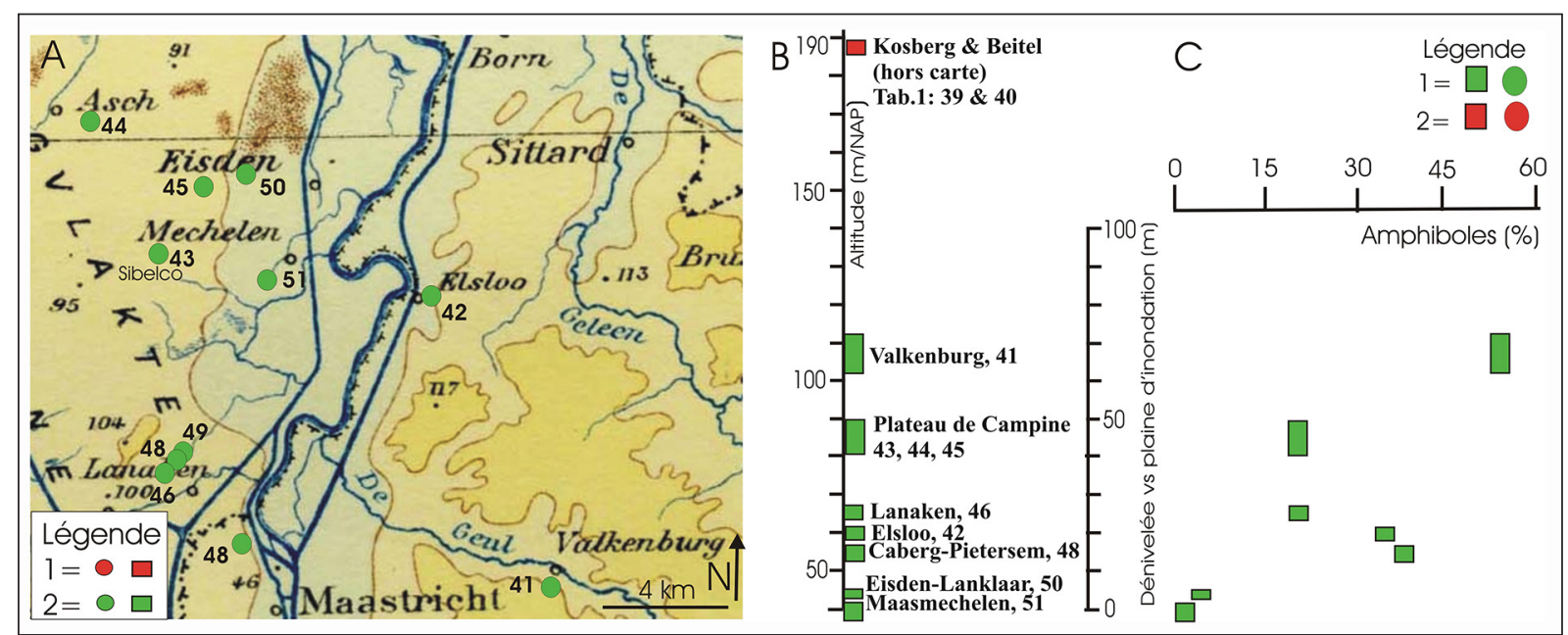

Figure 6. Répartition des hornblendes des Vosges dans des terrasses mosanes du Limbourg (Belgique et Pays Bas) selon Bustamante (1973). (A) Position géographique des sites de prélèvement. N.B. Les sites de Kosberg et de Beitel sont hors carte à l'est (Ostmaas). (B) Répartition stratigraphique des lbxt étudiés conformément au modèle de l'auteur (Bustamante, 1973). (C) Fréquence des hornblendes des Vosges dans les différents lbxt. Légende : 1= lbxt avec hornblende ; $2=1$ bxt sans hornblende. Les numéros des sites et terrasses sont ceux du Tableau 1 (annexe). 


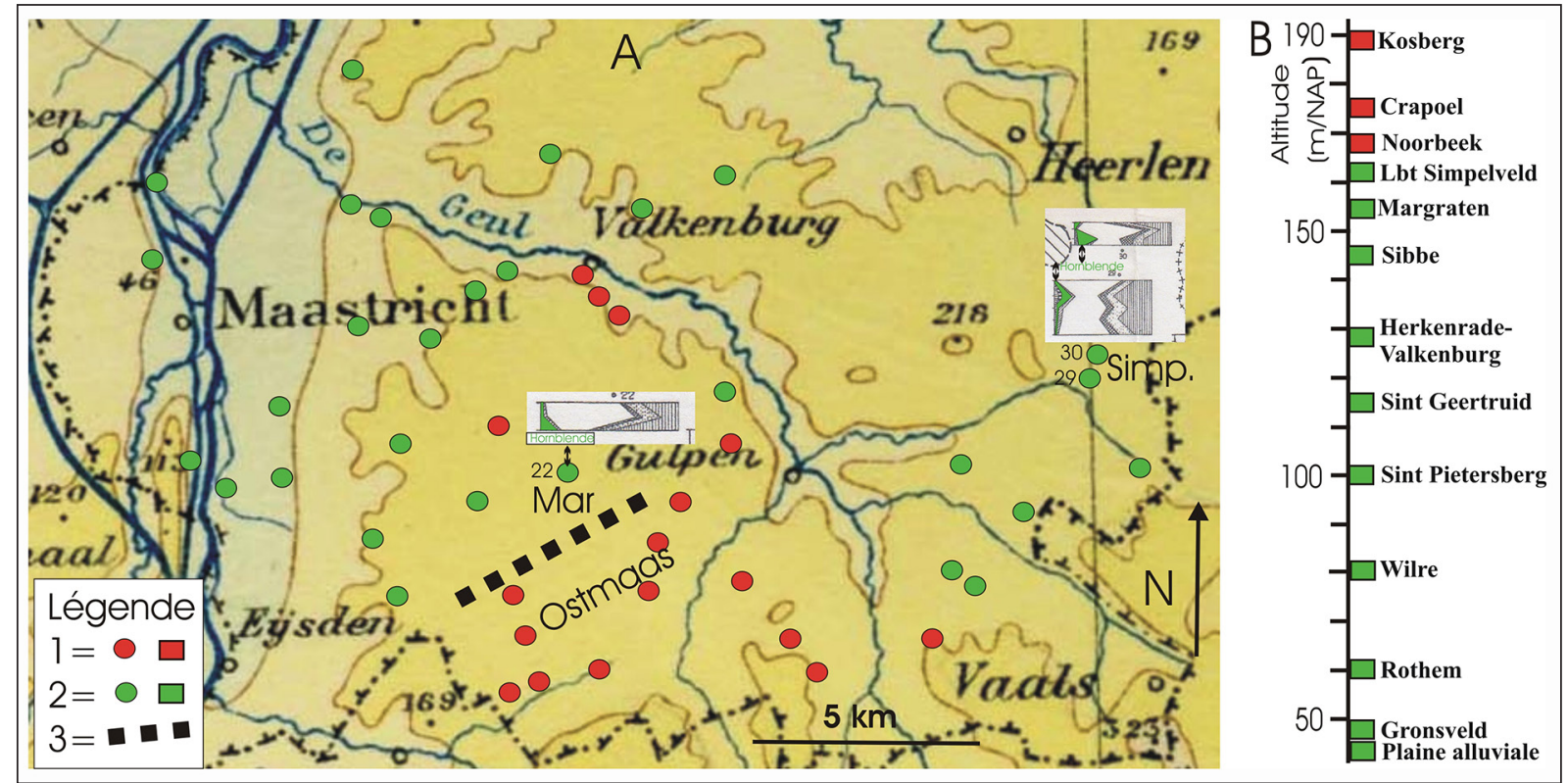

Figure 7. Répartition des hornblendes brunes et vertes dans des terrasses mosanes du Limbourg néerlandais selon Zonneveld (1949 : d'après la planche annexe du travail original). (A) Répartition géographique des sites étudiés. Légende : $1=1$ bxt sans hornblendes ; $2=1$ bxt avec hornblendes ; $3=$ crête de Banholt séparant l'Ostmaas (Hol, 1949) de la Westmaas. Abréviations : Simp= Simpelveld ; Mar= Margraten. Les diagrammes des lbxt de Margraten et de Simpelveld, sur lesquels les plages de hornblendes ont été mises en évidence en vert, ont été introduits en raison de leur intérêt particulier. (B) Répartition stratigraphique des hornblendes; les sites sont placés en fonction de l'altitude absolue de la base des cailloutis d'après Brueren (1945) ou Felder et Bosch (1989). Dans chaque site, plusieurs échantillons ont été traités et les résultats ne sont exprimés que sous forme de mini-graphiques si bien qu'aucun diagramme de fréquence n'a pu être dessiné. De plus, le calcul de la dénivelée par rapport à la plaine d'inondation pose problème dans l'Ostmaas en raison de la distance qui sépare la majorité des sites, de l'orientation différente de la Meuse (notamment dans l'Ostmaas), mais aussi de déformations tectoniques non quantifiées.

terrasses de la région de Givet, l'entrée en scène des amphiboles est clairement contrainte à $128 \mathrm{~m}$ $(\mathrm{d}+28 \mathrm{~m})$. Entre Hun (Yvoir sud) et Chemin d'Erpent (Namur Est), les amphiboles sont présentes entre 110 et $125 \mathrm{~m}$, mais leur absence n'est avérée qu'à 135 $140 \mathrm{~m}$ (d56 à Lives-sur-Meuse) et plus haut. Dans la Basse-Meuse liégeoise, elles entrent en scène $140 \mathrm{~m}$ sur le flanc droit (La Tombe/Dahlem : d74). Sur le flanc gauche aucune altitude maximale ne peut être fixée dans la mesure où tous les lbxt sont plus bas que $140 \mathrm{~m}$ et contiennent tous les amphiboles des Vosges.

En conséquence, si l'on accepte que l'entrée en scène des amphiboles dans les terrasses de la Meuse de Givet jusqu'au Limbourg représente la capture de la Meuse lorraine, on doit en déduire un basculement postérieur du sol vers l'Ouest de l'axe Namur-Basse Meuse. On notera ici qu'un tel basculement a été évoqué antérieurement pour la terrasse dite principale depuis Mouchamps (1933) ; il s'agit de T4 dans la Figure 8. Ce basculement simple a été transformé par Pissart (1974) en une déformation anticlinale dont l'axe se situe entre Huy et Liège. Toutefois, Juvigné et al. (ce fascicule et 2020b) ont montré que les raccords ayant conduit à la reconstitution de la dite terrasse sont inacceptables pour des raisons pétrographiques. Quant au basculement inhérent à la terrasse à amphibole de Margraten à Simpelveld (Zonneveld, 1949), il devrait relever de la tectonique de la Baie du Rhin inférieur.

Par ailleurs, l'intégration de la zone d'entrée en scène des amphiboles dans le modèle pose question. Entre Givet et le Limbourg, elle recoupe les terrasses de T4', T5 et T5'. On peut rappeler ici que Juvigné et al. (2013) ont montré que la terrasse dite principale de la Meuse (T4 sur la Figure 8) qui est rapportée en contre-pente dans la littérature (Clairbois, 1957, 1959 ; Pissart 1974 et Pissart et al., 1997a) présente en réalité une pente identique à celle de la plaine alluviale actuelle, au moins entre Seilles et Huy. Juvigné et al. (2020a et 2020b) montrent que les déformations tectoniques attribuées aux hautes terrasses entre Namur et Huy sont en contradiction avec les données pétrographiques propres aux cailloutis des mêmes terrasses. Enfin, l'étude récente de très hautes terrasses à Bois de 
des amphiboles des Vosges dans les terrasses mosanes

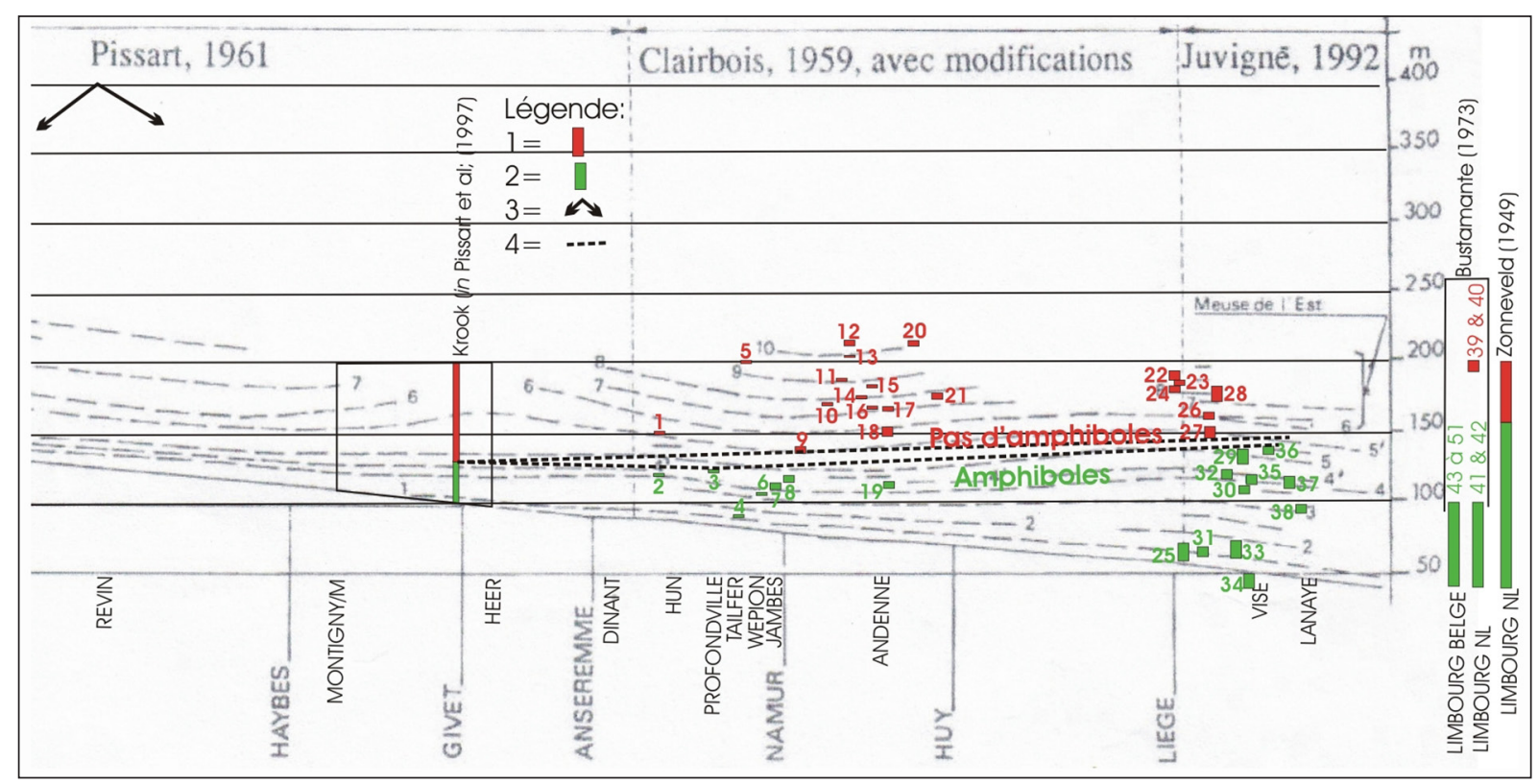

Figure 8. Répartition des amphiboles dans des lbxt depuis Givet jusque dans le Limbourg sur un extrait du dernier modèle en date des terrasses mosanes (Pissart et al., 1997a : Figure 2, p. 269). Légende : 1= cailloutis mosan sans amphiboles ; 2= cailloutis mosan avec amphiboles ; 3= ligne de crête du Massif de Rocroi ; 4= zone de transition entre les terrasses à amphiboles et celles qui en sont dépourvues. Explications : (1) les numéros attribués aux différents sites correspondent à ceux du tableau 1 (annexe) où les associations de minéraux denses transparents sont rassemblées ; (2) le graphique de référence de Krook a été établi par l'étude de lbxt répartis entre Montigny-sur-Meuse et Heer, et les altitudes relatives sont intégrées à Givet ; (3) dans le travail de Zonneveld (1949) les lambeaux sont répartis sur l'ensemble du Limbourg néerlandais, et nous avons intégré les résultats dans une colonne stratigraphique sans respect des distances, mais l'altitude du lbt de Margraten est déterminante (voir texte) ; (4) Bustamante (1973) a étudié des lambeaux de toutes les terrasses du Limbourg belge identifiées par Paulissen (1973), et aussi de quelques lambeaux du Limbourg néerlandais ; ses résultats sont présentés de façon intégrée dans deux colonnes stratigraphiques sans respect des distances.

Breux (Liège) évoque entre autres la possibilité d'un basculement transversal de la vallée de la Meuse liégeoise (Juvigné et Van Campenhout, 2020), et de ce fait jette un doute sur la fiabilité du modèle dans la Basse-Meuse qui est en révision par Paulissen et Juvigné (en préparation).

\section{B. À propos de l'altération des amphiboles}

La question de la possible disparition des amphiboles par altération prend ici toute sa pertinence. $\mathrm{La}$ littérature est très abondante en la matière et nous renvoyons à Bustamante (1973: p.79-96) qui en a fait un tour d'horizon détaillé au terme duquel on constate effectivement que les amphiboles ne font jamais partie des minéraux ultrastables, très stables, stables, voire semi stables. Dans le problème qui nous occupe ici, nous nous limiterons aux conclusions de Bustamante, puisque c'est lui qui a conclu à la disparition des hornblendes des Vosges par altération dans les terrasses anciennes de la Meuse, et qu'il a été suivi par tous les auteurs qui ont défendu l'âge pré-Quaternaire de la Meuse lorraine. L'expérience au terme de laquelle il a obtenu sa propre échelle d'altérabilité (p.82-84) est une attaque chimique par une solution de $\mathrm{HNO}_{3}$ $(14.4 \mathrm{~N})+\mathrm{HCl}(12.2 \mathrm{~N})$ de $\mathrm{pH}$ 2.54. L'auteur précise lui-même que de telles conditions n'existent pas dans la nature, mais que cela « permettait une accélération de l'attaque, c'est-à-dire une grande exagération du facteur temps. En déterminant le contenu en fer des solutions acides nous pouvions espérer d'établir une corrélation entre la corrosion et la disparition progressive des minéraux riches en fer. " À chaque lecteur son avis sur l'application de ce résultat à la disparition des amphiboles des Vosges dans les cailloutis.

Par ailleurs, de Ridder (in Cordier, 2004) montre la diminution des amphiboles sur les moyennes terrasses supérieures de la Moselle où elles restent cependant présentes. À une autre échelle de temps, dans son étude des minéraux denses transparents des terrains post-paléozoïques de la Belgique, Tavernier (1947) fait état de la présence de plusieurs pourcents d'amphiboles dans des terrains meubles 
marins ou continentaux de Flandre, et notamment de l'Éocène (Paniselien), de l'Oligocène (Tongrien Tg2a à Tg1d, Rupelien et Chattien), du Miocène (Anversien, Diestien et Scaldisien). Les amphiboles peuvent donc aussi résister à l'altération dans des matériaux détritiques pendant plusieurs dizaines de millions d'années.

\section{C. À propos de la fréquence des amphiboles dans les frottis}

Les considérations qui précèdent montrent qu'il n'y a pas de schéma simple pour expliquer la conservation ou l'altération des amphiboles par voie chimique, en raison des conditions environnementales très variables qu'elles ont subies. On peut aussi s'étonner de différences de fréquence importantes des amphiboles entre deux terrasses successives, voire entre des échantillons d'un même lbt (constatation faite notamment à Haccourt, Herstal et Lixhe) ou encore de l'augmentation éventuelle des fréquences avec l'éloignement par rapport aux Vosges.

À ce sujet, on peut ajouter que tous ceux qui ont trié des minéraux à l'aiguille sous la loupe, et qui ont légèrement pressé une amphibole plutôt que de la faire glisser sur le porte-objet ont constaté qu'elle se brise aisément. Ceci implique qu'en cours d'extraction de minéraux, les agitations abusives (tamisage par secousses plutôt que par courant d'eau) peuvent dans un premier temps augmenter la fréquence des amphiboles, et ensuite les faire disparaître lors d'un tamisage final. Il faut aussi se souvenir que les cailloux de granites sont très rares dans les cailloutis parce qu'ils se sont désagrégés sous l'effet des tensions physiques (notamment chocs entre cailloux, dilatation/contraction par variations de température, voire d'humidité) ; un échantillon qui a empiété sur un granite décomposé (voire indécelable) peut donc contenir davantage d'amphiboles que son voisin, même immédiat. En conclusion, toutes ces remarques invitent à ne pas accorder aux variations de fréquences des amphiboles une importance qu'elles ne méritent pas.

\section{Des faiblesses dans l'argumentation du dernier modèle en date dans la région de la capture de la Meuse lorraine}

Si l'origine du réseau hydrographique dans la zone concernée remonte à l'émersion post-crétacée
(Harmand et Cordier, 2012), le problème de la traversée du Massif de Rocroi par la Meuse, ne se pose effectivement que depuis d'Omalius d'Halloy (1842) et de façon de plus en plus ciblée avec Rutot (1897) et Dolfuss (1900) qui débattent du problème à l'altitude du plateau de Rocroi (400 m). Nordon (1928) propose que la Meuse lorraine aie transité dans les vallées de la Vrigne et de la Goutelle inférieure avant de traverser le col de Meillier-Fontaine (310-315 m) et de poursuive son cours par le Val de Faux. Pissart $(1961,1974)$ et Pissart et al. (1997) se basent sur la présence de kieseloolithes dans le dépôt fluviatile du col de Meillier-Fontaine (310-315 m) pour l'attribuer à la Meuse lorraine, puis faire capturer celle-ci à Nouzonville par la Meuse Dinant et la prolonger en Belgique par la Traînée mosane dont l'âge pliocène voire mio-pliocène est communément admis. Dans la mesure où l'entrée en scène des amphiboles des Vosges dans les terrasses mosanes en Belgique a lieu à un niveau nettement inférieur à la Traînée mosane, on fait face à une incompatibilité majeure. Dans ces conditions, on peut aussi mettre en exergue des points faibles de l'argumentation qui fait consensus depuis plus d'un demi-siècle, voire un siècle, concernant la région des captures entre Sedan, Charleville et Revin (Figure 9).

\section{La Meuse lorraine est-elle passée à Meillier-} Fontaine?

Bustamante et Voisin (1975) montrent que les matériaux qui composent le dépôt de Meillier-Fontaine sont uniquement d'origine ardennaise. Pissart (1974) n'en tient pas compte, mais la question est débattue entre autres par les trois chercheurs concernés pendant la discussion de l'excursion consacrée aux terrasses de la Meuse (Macar, 1974). Bustamante (1995) écrit que c'est « un petit cours d'eau » qui a dû passer à Meillier-Fontaine, excluant de façon implicite le passage de la Meuse lorraine par la vallée de Gespunsart (Vrigne et Goutelle). Pissart et al. (1997a) réagissent en mettant en exergue la ressemblance de l'association des minéraux denses transparents d'une part du cailloutis de Meillier-Fontaine, et d'autre part des sables miocènes d'Opgrimbie (Limbourg néerlandais).

\section{La Meuse lorraine est-elle passée par la vallée de Gespunsart?}

Bustamante et Voisin (1975) ont trouvé des hornblendes des Vosges dans le cailloutis supérieur 


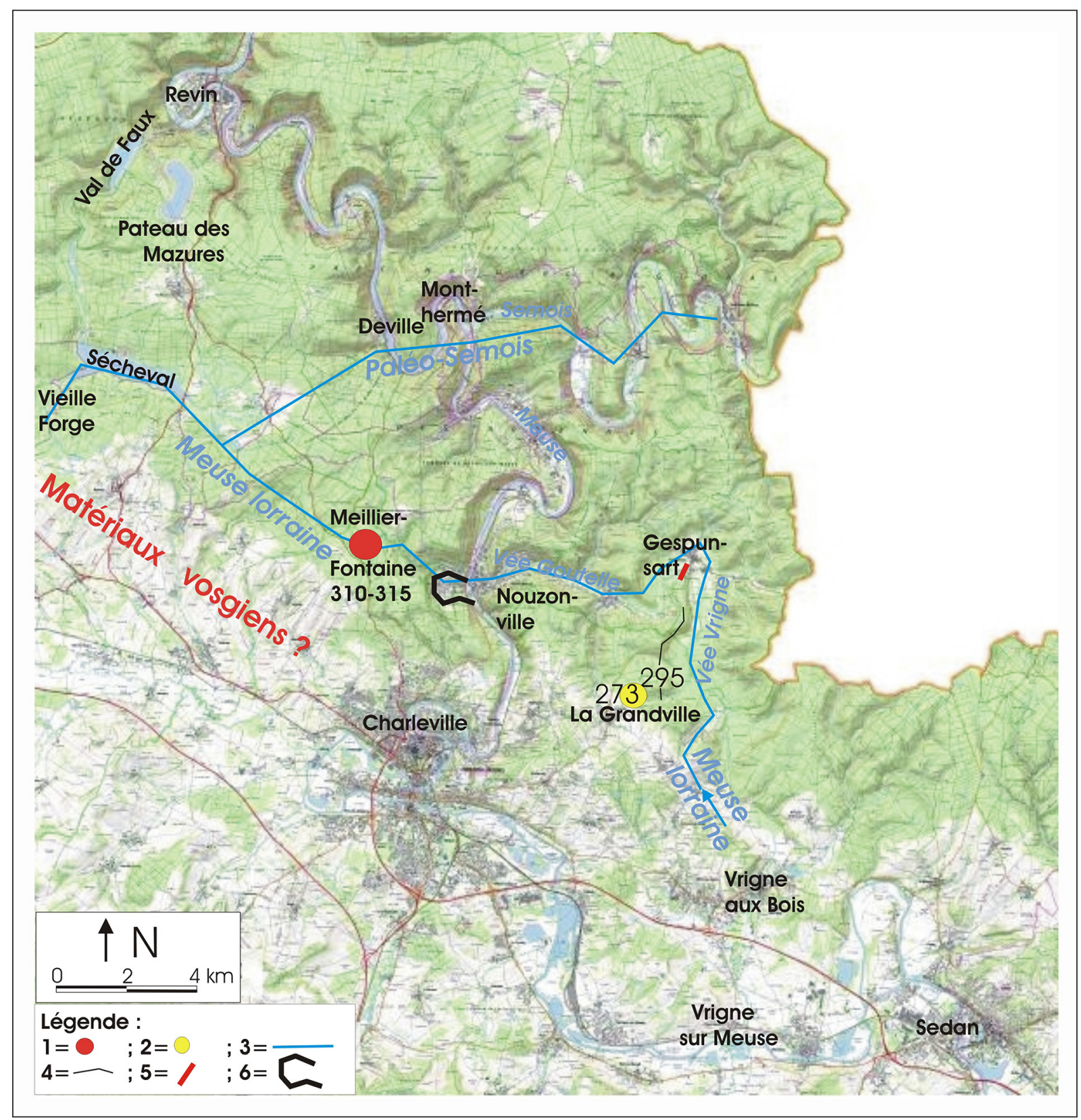

Figure 9. La zone des faiblesses de l'argumentation concernant la capture de la Meuse lorraine. (A) Localisation de sites évoqués dans le texte. Légende : $1=$ cailloutis de Meillier-Fontaine 310-315 m;2= cailloutis de La Grandville $(273 \mathrm{~m}) ; 3=$ cours supposés de la Meuse lorraine et de la paléo-Semois d'après Pissart et al. (1997a) ; 4= ligne de crête séparant la carrière de La Grandville de la vallée de la Vrigne ; 5=localisation de la coupe de la Figure 10 ; $6=$ méandre recoupé de Nouzonville.

de la carrière de La Grandville (à $273 \mathrm{~m}$ ), ce qui atteste le passage de la Meuse lorraine à cet endroit (Figure 9). Par ailleurs, cela exclut qu'à la même altitude, ce cours d'eau ait pu se trouver dans la vallée de Gespunsart dont il est séparé par une crête, qui est encore actuellement à $295 \mathrm{~m}$.

Macar (1945b) produit une étude géomorphologique de la vallée de Gespunsart, dans laquelle il met en exergue les difficultés d'y faire passer tant la Chiers que la Meuse lorraine, mais néanmoins il conclut :
« À première vue invraisemblable, cette hypothèse (NDR: la Meuse lorraine), après examen, se montre de loin la meilleure ». De plus l'auteur précise : «...l'ancienne vallée se trouve très vraisemblablement à 35 à $40 \mathrm{~m}$ au-dessus de la Meuse actuelle, et en tous cas à moins de 50 à $60 \mathrm{~m}$ de celle-ci. » Pissart (1961) interprète le replat du cimetière de Gespunsart comme un lbt de la Meuse lorraine.

Voisin (1967) décrit une excavation temporaire qui a été ouverte sur le replat qui domine le cimetière à 
$206 \mathrm{~m}$ (Saint-Lieu ; Figure 10) où il a vu une nappe de « ...quartzites bien roulés... dans des argiles rougeâtres... qui semblent provenir ... des niveaux supérieurs de la longue colline qui fut le pédoncule du méandre mosan » (sommet à $317 \mathrm{~m}$ ). Ce cailloutis recouvre des sables dont les fossiles sont attribués au Jurassique. Il n'est nulle part question d'éléments vosgiens dans ces terrains.

Observations personnelles. Dans le cimetière, il n'y a que des éléments schisteux dans les terres des tombes ; il devrait s'agir de remblais étalés pour assurer l'horizontalité du cimetière. Nous avons eu l'opportunité d'examiner une excavation réalisée pour installer l'entreprise Mécano-soudure Degembes au bord inférieur du cimetière; cela nous a permis de prolonger la coupe précitée de Voisin (Figure 10). L'affleurement présente dans sa partie inférieure un cailloutis sur $60 \mathrm{~cm}$ d'épaisseur, mais sa base n'a pas été atteinte, il est surmonté de sable limoneux finement stratifié, puis vers le haut, les structures sédimentaires ont disparu sous l'action de la pédogenèse. Le gravier est composé essentiellement d'éléments provenant de roches paléozoïques; pour un seau d'échantillon: (1) on ne trouve que des quartzites anguleux dans la classe de 32 à $64 \mathrm{~mm}$; (2) dans la classe de 16 à $32 \mathrm{~mm}$ (Figure 5), on trouve : (i) 62 galets présentant un émoussé marin dont 29 quartz, 29 quartzites et 4 grès ; (ii) 59 cailloux présentant une ébauche d'émoussé dont 2 quartz, 31 quartzites, 21 schistes et 5 grès ; (iii) 34 cailloux anguleux dont 8 quartz, 19 quartzites et 7 schistes. Les minéraux denses de la fraction sableuse comprise entre 75 et $425 \mu \mathrm{m}$ consistent $97 \%$ de minéraux indéterminables (essentiellement opaques et quelques débris rocheux) et seulement $3 \%$ de minéraux transparents avec dans l'ordre de fréquence : tourmaline $(53,7$ $\%$ ), zircon $(31,3 \%)$, rutile $(9,7 \%)$, épidote $(2,2 \%)$ et staurotide $(1,5 \%)$. Par comparaison avec le dépôt mosan de la carrière de La Grandville (cf. supra), un tel cailloutis ne peut évidemment appartenir à la Meuse lorraine.

Voisin (1979) rapporte une description simplifiée de six forages industriels profonds exécutés par percussion jusqu'au bedrock dans les vallées de la Vrigne et de la Goutelle, ce qui nous permet de dessiner la coupe de la Figure 11. L'auteur fait remarquer la difficulté d'identifier les éléments à l'état concassés, et il ne reconnait que des apports de versants et de cônes de déjections des affluents, tandis que les alluvions de la rivière qui a transité par ces deux vallées n'ont pu être identifiées. Il faut insister sur le fait que l'auteur écrit : «C'est là en effet au sondage de la Lutinière, que l'altitude maximum du bedrock est relevée avec 179,40 mètres. » Néanmoins, les forages 2-3 et 4 (Figure 11) n'ont apparemment pas été pris en compte par Pissart et al. (1998) pour reconstituer le profil de la « Base des alluvions de la Meuse lors de l'abandon du cours de Gespunsart. »

En conclusion, la présence du dépôt fluviatile de Meillier-Fontaine (310-315 m) ne peut s'expliquer que par l'action d'un cours d'eau qui débouchait de la vallée de Gespunsart. Ce devait être un "petit cours d'eau » aux alluvions ardennaises, ce qui fait penser à la Chiers plutôt qu'à la Meuse lorraine. L'accumulation des dépôts de versants et de cônes de déjections dans les vallées de la Vrigne et de la Goutelle n'a pu commencer qu'au moment où le cours d'eau en transit a été capturé, et ce ne peut être que lorsqu'il passait à $180 \mathrm{~m}$ (ou un peu plus) à Gespunsart. Dans ces conditions, on comprend mal le profil attribué par Pissart et al. (1998 : p.34, Figure 5) à la Meuse lorraine au moment de sa disparition de la vallée de Gespunsart. La mise en doute de ce profil qui devrait faire place à un autre nettement plus élevé, rend indéfendable l'hypothèse de la capture de

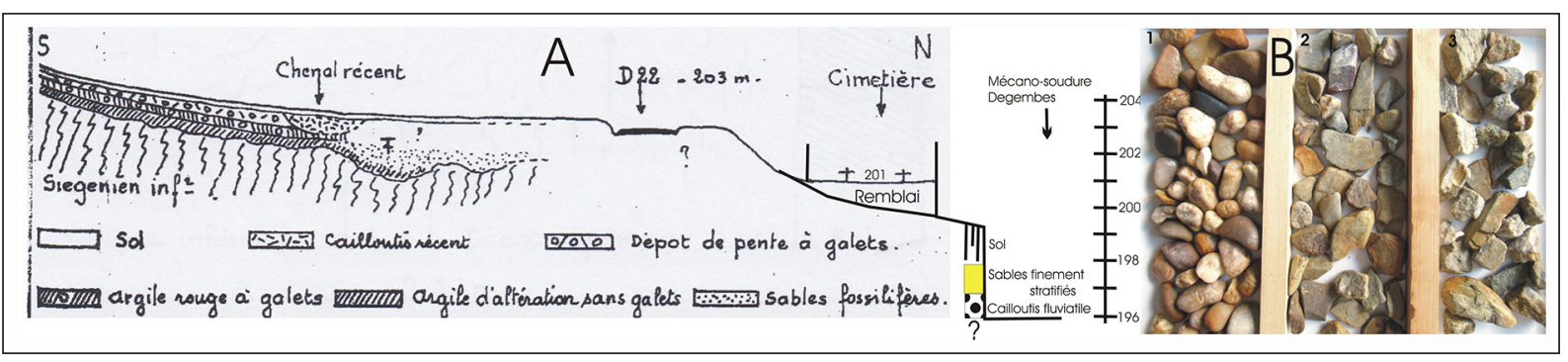

Figure 10. Coupe de Saint-Lieu et du cimetière de Gespunsart (localisation de la coupe sur la Figure 9). (A) La coupe de l'excavation de Saint Lieu (Voisin, 1967, p.77, figure 1, avec ajout du site excavé pour l'installation de l'entreprise Degembes (Mécano-soudure). (B) Le gravier grossier (16-32 mm) du cailloutis de Gespunsart (Entreprise Degembes, Mécano-Soudure) : (1) galets à émoussé marin, essentiellement quartz et quartzite ; (2) cailloux avec ébauche d'émoussé, uniquement débris du socle paléozoïque ; (3) cailloux anguleux, uniquement débris du socle paléozoïque. 


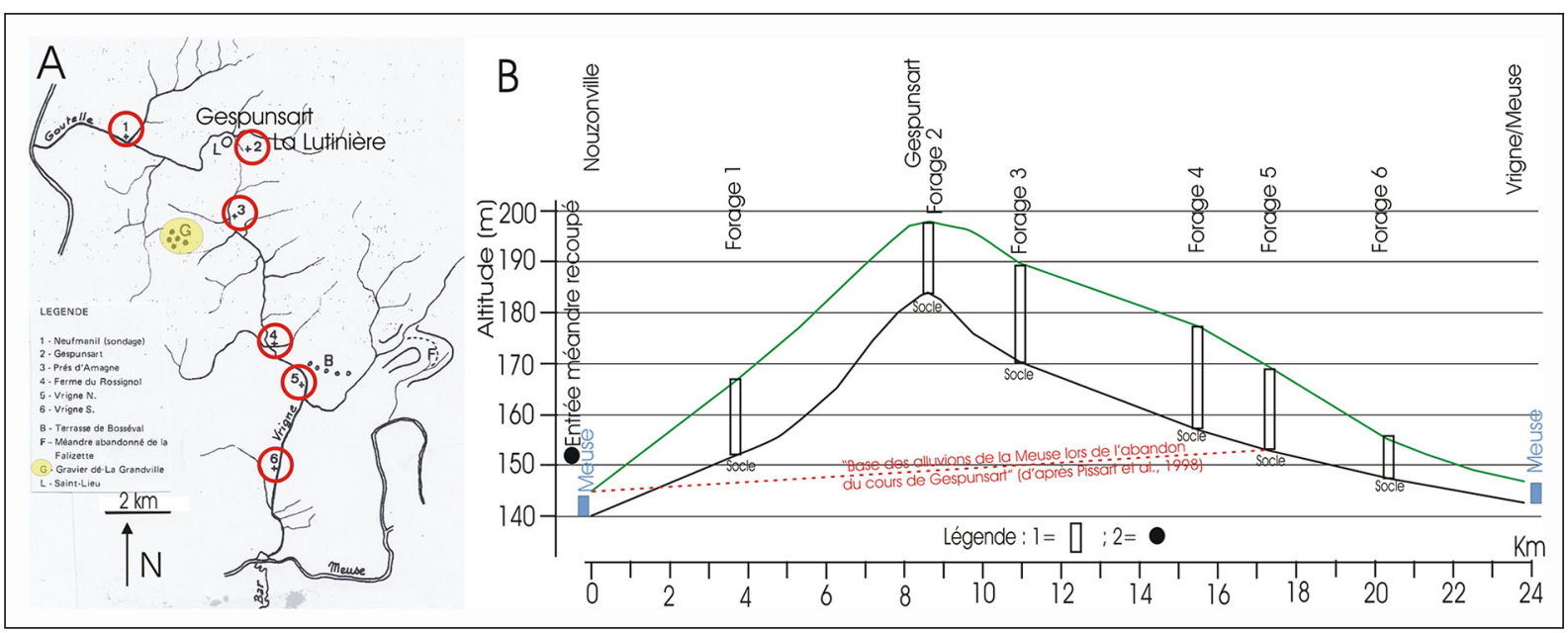

Figure 11. (A) Localisation des forages industriels exécutés dans les vallées de la Vrigne et de la Goutelle. Légende : 1=Neufmanil ; 2=La Lutinière/Gespunsart ; 3= Pré d'Amagne ; 4= Ferme du Rossignol ; 5=Vrigne N ; 6=Vrigne $\mathrm{S}$. ; $\mathrm{B}=$ terrasse de Bosseval ; $\mathrm{F}=$ méandre abandonné de la Falizette ; $\mathrm{G}=$ gravier de La Grandville ; $\mathrm{L}=$ Saint-Lieu. (B) Coupe géologique le long de la Vrigne et de la Goutelle ; Légende : 1= forage à travers la couverture meuble jusqu'au bedrock ; 2 = cailloutis fluviatile à l'entrée du méandre abandonné de Nouzonville (Pissart et al., 1997b) ; trait vert= profil du fond de vallées ; trait noir= profil du bedrock.

la Meuse lorraine par la Bar à Vrignes-sur-Meuse. À ce sujet, on peut rappeler que Macar (1945a) avait publié un article intitulé « L'étrange capture de la Meuse par la Bar » proposée par Nordon (1928). Néanmoins, Pissart (1960) a défendu cette hypothèse dans un article où il développe lui-même les objections auxquelles elle est confrontée, et tout en restant constamment dans le doute, il conclut à la capture qui depuis lors est devenue une certitude sans avoir jamais été démontrée.

Dans ce contexte, le méandre abandonné de Nouzonville ne peut plus être attribué à la Bar, et ses alluvions décrites par Pissart et al. (1998) ne peuvent être celles de la Goutelle décrites par Voisin (1967). Dès lors, l'absence d'amphiboles des Vosges dans le cailloutis de la très basse terrasse du méandre située moins de $6 \mathrm{~m}$ plus haut que la plaine d'inondation (Pissart et al., 1997b) peut s'expliquer par comparaison avec le graphique de référence des terrasses à Givet (Pissart et al., 1997a\&b), par sa postériorité par rapport à la capture de la Moselle à Toul.

\section{Réflexions sur la présence des kieseloolithes et des amphiboles des Vosges}

Pissart (1961) a utilisé les traces de kieseloolithes présentes à la fois dans des dépôts élevés de la région des captures en France et dans la Traînée mosane en Belgique pour défendre leur corrélation. Des remarques s'imposent : (1) dans le dépôt de Meillier-Fontaine, il y a à la fois des traces de kieseloolithes et d'amphiboles, tandis que dans la Traînée mosane les amphiboles sont inconnues (cf. supra) ; (2) Macar (1945a), au terme de son étude détaillée des dépôts $O n x$ en Belgique écrit : «... la seule présence de kieseloolithes dans un gisement ne possède plus, comme moyen de corrélation, qu'une valeur nettement limitée. »; (3) par ailleurs, la présence d'amphiboles dans le dépôt de La Granville atteste que ce minéral peut être conservé dans un cailloutis mosan très ancien ( $c f$. l'altération des amphiboles).

\section{Les attentes dans la vallée de la Sormonne}

Rutot (1897) défend l'idée qu'à l'aval de Mézières, la Meuse lorraine poursuivait son cours par la vallée actuelle de la Sormonne, contournait le massif paléozoïque d'Entre Sambre et Meuse puis empruntait l'axe Sambre Meuse actuel. De la même façon, Pissart $(1960,1961)$ propose l'hypothèse selon laquelle la Meuse lorraine s'écoulait autrefois vers la Seine via la vallée de la Sormonne. Néanmoins, aucun travail ne fait encore état de la présence ou de l'absence de matériaux vosgiens dans la vallée notamment de la Haute Sormonne, et plus particulièrement aux portes de Charleville (revoir la Figure 9).

\section{VI. À PROPOS DE L'ÂGE DE LA CAPTURE DE LA MEUSE LORRAINE}

D'une part, en matière de chronologie des terrasses, les sites de référence se trouvent essentiellement 
dans le Limbourg néerlandais (Felder et Bosch, 1989 ; Van den Berg, 1996). Toutefois, il faut remarquer que dans ces modèles, les altitudes des terrasses sont transférées dans un profil de référence passant par Maastricht et que Felder et Bosch (1989) considèrent la base des cailloutis, tandis que Van den Berg (1996) travaille sur le sommet des mêmes dépôts.

Par ailleurs, dans l'opération de transfert des altitudes, chaque auteur a disposé d'une marge de liberté en raison (revoir la Figure 7) : (1) du changement d'orientation du cours d'eau ; (2) de l'éloignement des terrasses par rapport au tronçon Maastricht-Maaseik (revoir les Figures 1 et 7) ; (3) de déformations du sol dans le Limbourg néerlandais qui ne sont pas quantifiées.

D'autre part, Pissart et al. (1997a) proposent les corrélations suivantes pour les trois terrasses recoupées par la zone d'entrée en scène des amphiboles : (1) $\mathrm{T} 4{ }^{\prime}=\mathrm{T}$. de Sint Geertruid- $2=0,89 \mathrm{Ma}$ sensu Felder et Bosch (1989) ; (2) T5 (dite T. de Bombaye-Wonck $=\mathrm{T}$. de La Tombe $/$ Dahlem $)=\mathrm{T}$. de Sint Geertruid-1=1,05 Ma sensu Felder et Bosch (1989) ; (3) T5'= T. de Valkenburg-1 (NDR : qui contient des amphiboles $)=\mathrm{T}$. de Hognée $(N D R$ : qui ne contient pas d'amphiboles $)=1,3 \mathrm{Ma}$ sensu Felder et Bosch (1989). L'âge des mêmes terrasses est systématiquement plus ancien chez Van den Berg (1996) : (1) T. de Sint Geertruid-2, 1,09 Ma (T4') ; (2) T. de Sint Geertruid-1, -1,28 Ma (T5) ; (3) T. de Valkenburg-1, 1,57 Ma.

Par ailleurs, pour éviter les incertitudes relatives à la fiabilité du modèle de terrasse précité, si l'on confronte directement l'altitude de la limite des amphiboles aux portes de Maastricht (Lbt de Lixhe : $140 \mathrm{~m}$; Figure 9) avec le profil de référence à Maastricht de Van den Berg (1996), on lit à $139 \mathrm{~m}$ : T. de Valkenburg-1, 1,57 Ma.

Il reste à se souvenir que Zonneveld (1949) montre que les hornblendes brunes et vertes sont présentes dans les lbxt de Margraten et de Simpelveld et que Felder et Bosch (1989) et Van den Berg (1996) en font des terrasses distinctes. À titre d'exemple, ce dernier auteur en rapporte les âges suivants : T. de Margraten, $159 \mathrm{~m}$ à Maastricht 1,87 Ma ; (2) T. de Simpelveld-1, 175 m à Maastricht 2,14 Ma. La dispersion de l'ensemble des valeurs à Maastricht est pour le moins interpellante.
En conclusion, il est évident qu'en fonction de la distribution des amphiboles dans les terrasses mosanes, la capture de la Meuse lorraine a dû avoir eu lieu nécessairement avant la formation de la terrasse T4 (dite principale) qui domine la plaine alluviale actuelle d'une trentaine de mètres à Namur et s'en écarte dans la Basse-Meuse pour atteindre une dénivelée d'une soixantaine de mètres à Visé. Les estimations d'âge de cette dernière s'accordent à placer sa formation pendant la transition du Pléistocène inférieur au Pléistocène moyen : 0,7 Ma sensu Felder et Bosch (1989) ; 0,78 Ma sensu Van den Berg (1996) ; 725 \pm 120 ka/CNR pour le lambeau de Romont-2/Bassenge sensu Rixhon et al. (2011).

\section{CONCLUSION}

Aussi longtemps que l'on ne trouve pas une provenance autre que les Vosges pour les amphiboles vertes et/ou brunes qui sont présentes dans les sables de terrasses de la Meuse en Belgique, la relation entre leur entrée en scène et la capture de la Meuse lorraine sera pertinente. À la porte du Limbourg, la terrasse qui contient le lbt à amphiboles le plus élevé (Lixhe [b134; s140; d80]) est à la même altitude que la T. de Valkenburg-1, datée de 1,57 Ma, ce qui devrait placer la capture de la Meuse lorraine dans la seconde moitié du Pléistocène inférieur.

Cette nouvelle hypothèse relative à la capture de la Meuse lorraine impose de toute évidence de revisiter la région des captures de la Semois et de la Meuse lorraine entre Sedan et Revin où il est apparu qu'en accordant la préséance aux données sédimentologiques locales, un nouveau modèle de l'évolution du réseau hydrographique pourrait être proposé.

\section{BIBLIOGRAPHIE}

Beiner, M., Harmand, D., Cordier, S. \& Occhietti, S. (2009). Les minéraux lourds des alluvions quaternaires du bassin de la Moselle : nouvelles données. Quaternaire, 20, 63-80.

Bourguignon, P. (1954). Les sables des Hautes Fagnes. Annales de la Société géologique de Belgique, 77 : B201-241.

Brueren, J.W.R. (1945). Het terrassenlandschap van Zuid-Limburg. Med. Geol. Stichting, Serie C-VI-1.

Bustamante-Santa Cruz, L. (1973). Les minéraux lourds des alluvions sableuses du bassin de la Meuse. Thèse 
des amphiboles des Vosges dans les terrasses mosanes

de doctorat, Katholieke Universiteit te Leuven, Faculteit der Wetenschappen, 355 p., Leuven.

Bustamante-Santa Cruz, L. (1976). L’évolution plio-pléistocène du bassin mosan d'après ses minéraux lourds. Revue de Géographie physique et de Géologie dynamique, 8(4) : 291-300.

Bustamante-Santa Cruz, L. (1986). Aperçu sur l'évolution actuelle et plio-pléistocène du bassin mosan d'après ses minéraux lourds. Annales des Mines de Belgique, 9-10, 835-839.

Bustamante-Santa Cruz, L. (1995). Contribution to the petrographical characterization of the Quaternary Meuse alluvia river terraces by way of heavy mineral analyses. Neues Jahrbuch für Mineralogie, 12, 529-552.

Bustamante-Santa Cruz, L. \& Voisin, L. (1975). La capture de la Meuse lorraine par la Meuse de Dinant, remise en question partielle basée sur l'analyse des minéraux lourds. Ministère des Affaires économiques, Administration des Mines, Service géologique de Belgique, 17 pages.

Clairbois, A.-M. (1957). L'évolution du cours de la Meuse entre Liège et Anseremme au cours du Quaternaire. Université de Liège, Laboratoire de Géographie physique. Mémoire de licence inédit. 175 p.

Clairbois, A.-M. (1959). L'évolution du cours de la Meuse entre Liège et Anseremme au cours du Quaternaire.

Cordier, S. (2004). Les niveaux alluviaux quaternaires de la Meurthe et de la Moselle entre Baccarat et Coblence: étude morphosédimentaire et chronostratigraphique, incidences climatiques et tectoniques. Université Paris 12, 455 pp.

Davis, W.M. (1895). La Seine, la Meuse et la Moselle. Annales de Géographie, 4, 25-49.

Demoulin, A. (1987). Les sables oligocènes du Plateau des Hautes Fagnes : une synthèse. Bulletin de la Société belge de Géologie, de Paléontologie et d'Hydrographie, 96, 81-90.

D'Omalius d'Halloy, J.-B. (1842). Coup d'œil sur la géologie de la Belgique. Bruxelles, p.3.

Dollfus, G. (1900). Relation entre la structure géologique du Bassin de Paris et son hydrographie. Annales de Géographie, IX, 313-339.

Felder, W.M. \& Bosch, P.W. (1989). Geologische kaart van Zuid-Limburg en omgeving. Afzettingen van de Maas. Rijks Geologische Dienst, Haarlem, NL.

Harmand, D. (1992). Histoire de la vallée de la Meuse lorraine. Presse universitaire, Nancy, $146 \mathrm{p}$.

Harmand, D. \& Cordier, S. (2012). The Pleistocene terrace staircase of the present and past rivers downstream from the Vosges (Meuse and Moselle catchments). Netherlands Journal of GeosciencesGeologie and Mijnbouw, 91(1/2), 91-109.

Hol, J. (1949). Geomorfologie. Handboek der Geographie van Nederland, I, 240-319.

Juvigné, E. (1978). Les minéraux denses transparents des lœss de Belgique. Zeit. Für Geomorphologie, $22,68-88$.
Juvigné, E. (1979). L’encaissement des rivières ardennaises depuis le début de la dernière glaciation. Zeitschrift für Geomorphologie, 23, 291-300.

Juvigné, E. (1993). Contribution à la téphrostratigraphie du Quaternaire et son application à la géomorphologie. Mémoires pour servir à l'Explication des Cartes Géologiques et Minières de la Belgique, Bruxelles, volume 36, $66 \mathrm{pp}$.

Juvigné, E., Dimodica, K., Houbrechts, G. \& Pirson, S. (2013). La pente longitudinale de la terrasse principale de la Meuse revisitée de Seilles à Huy (Belgique). Bulletin de la Société géographique de Liège, 61, 69-80.

Juvigné, E. \& Van Campenhout, J. (2020). Un site de référence de très hautes terrasses fluviales dans la zone de confluence Ourthe-Meuse à Bois de Breux (Liège). Bulletin de la Société Royale des Sciences, 89, 15-38. https://doi.org/10.25518/0037-9565.9426 Juvigné, E., Houbrechts, G. \& Van Campenhout, J. (2020a). A propos d'un changement pétrographique majeur dans les terrasses de la Meuse entre Namur et Huy (Belgique) : de la Meuse de Dinant à la capture de la Semois. Ce fascicule.

Juvigné, E., Houbrechts, G. \& Van Campenhout, J. (2020b). À propos d'un changement pétrographique majeur dans les terrasses de la Meuse entre Namur et Huy (Belgique) : de la Meuse de Dinant à la capture de la Semois. Rapport de travail. Site institutionnel Orbi de l'Université de Liège : http://hdl.handle. net $/ 2268 / 244521$

Juvigné, E., Bruni, Y. \& Hatert, F. (2020c). À propos de la détermination des chloritoïdes en frottis : le cas des alluvions de la Meuse et d'affluents ardennais. Rapport de travail. Site institutionnel Orbi de l'Université de Liège : http://hdl.handle.net/2268/245521

Krook, L. (1993). Heavy minerals in the belvedere deposits. In Maastricht Belvédère II. Mededelingen Rijks Geologische Dienst, 47, 25-30.

Leake, B.E. and the members of the Subcommittee on amphiboles of the International Mineralogical Association on new minerals and mineral names, 1997. Nomenclature of amphiboles. European Journal of Mineralogy, 9, 623-651.

Macar, P. (1945a). L'étrange capture de la Meuse par la Bar. Annales de la Société géologique de Belgique, 68, B198-213.

Macar, P. (1945b). La valeur, comme moyen de corrélation, des cailloux d'oolithe silicifiée et l'origine des graviers dits « Onx » des Hautes-Fagnes. Bulletin de la Société belge de géologie, de Paléontologie et d'Hydrologie, LIV, 214-253.

Macar, P. (1945b). L'étrange capture de la Meuse par la Bar. Annales de la Société géologique de Belgique, 68, B198-213.

Macar, P. (1974). La Traînée mosane aux environs de Liège. Excursion de l'excursion de mercredi 11 septembre 1974. In, L'évolution quaternaire des bassins fluviaux de la Mer du Nord méridionale, 
Centenaire de la Société géologique de Belgique, 1974, 291-296.

Mantniecks, P. (1955). Atlas classique. Institut cartographique P. Mantniecks, Bruxelles.

Mouchamp,s L. (1933). Les terrasses de la Meuse et de la Sambre. Annales de la Société géologique de Belgique, 82, B 213-233.

Nordon, R. (1928). Sur deux anciens cours de la Meuse ardennaise. Bulletin de l'Association des Géographes français, 23-24, 40-44.

Paulissen, E. (1973). De Morfologie en de kwartairstratigrafie van de Maasvallei in Belgisch Limburg. Verhandelingen van de Koninklijke Vlaamse Academie voor Wetenschappen, Letteren en Schone Kunsten van Belgie, klasse der Wetenschappen, jg. 35, $\mathrm{n}^{\circ}$ 127, Brussel.

Pirson, S., Baele, J.-M., Balescu, S., Haesaerts, P., Juvigné, E., Meijs, E. \& Spagna, P. (2018). Green amphibole distribution as a stratigraphic tool in loess sequences from Belgium: a review. Quaternary International, 485, 183-198.

Pissart, A. (1960). Le méandre recoupé du Bois de la Falizette et la capture de la Meuse par la Bar. Annales de la Société géologique de Belgique, 83, 115-125.

Pissart, A. (1961). Les terrasses de la Meuse et de la Semois. La capture de la Meuse de la Meuse lorraine par la Meuse de Dinant. Annales de la Société géologique de Belgique, 84, 1-108.

Pissart, A. (1974). La Meuse en France et en Belgique. Formation du bassin hydrographique. Les terrasses et leurs enseignements. In, L'évolution quaternaire des bassins fluviaux de la Mer du Nord méridionale, Centenaire de la Société géologique de Belgique, 1974, 105-131.

Pissart, A., Harmand, D. \& Krook, L. (1997a). L'évolution du cours de la Meuse de Toul à Maastricht depuis le Miocène: corrélations chronologiques et traces des captures de la Meuse lorraine d'après les minéraux denses. Géographie physique et Quaternaire, 51, 267-284.

Pissart, A., Krook, L. \& Harmand, D. (1997b). La capture de l'Aisne et les minéraux denses des alluvions de la Meuse dans les Ardennes. Comptes Rendus de l'Académie des Sciences de Paris. Sciences de la Terre et des Planètes. 325, 411-417.
Pissart, A., Krook, L. \& Harmand, D. (1998). Modifications du tracé de la Meuse dans la région de Charleville-Mézières en liaison avec les captures de l'Aisne et de la Moselle. Bulletin de la Société géographique de Liège, 35, 29-39.

Rixhon, G., Braucher, R., Bourlès, D., Siame, L., Boby, B. \& Demoulin, A. (2011). Quaternary river incision in NE Ardennes (Belgium)- Insights from ${ }^{10} \mathrm{Be} /{ }^{26} \mathrm{Al}$ dating of river terraces. Quaternary Geochronology, 6, 273-284.

Rutot, A. (1897). Les origines du Quaternaire de la Belgique. Bulletin de la Société belge de Géologie, de Paléontologie et d'Hydrologie, XI, 1-140.

Tavernier, R. (1947). Aperçu sur la pétrologie des terrains post-paléozö̈ques de la Belgique. La géologie des terrains récents dans l'ouest de l'Europe, p.69-90.

Voisin, L. (1967). Entre Vrigne et Goutelle, quelques faits nouveaux. Bulletin de la Société d'Histoire naturelle des Ardennes, 57, 71-81.

Voisin, L. (1979). Le couloir Vrigne-Goutelle. Etat de la question en 1979. Bulletin de la Société d'Histoire Naturelle des Ardennes, 89, 24-31.

Van den Berg, M.W. (1996). Fluvial sequences of the Maas: a 10 Ma record of neotectonics and climate change at various time-scales. $\mathrm{PhD}$ Thesis, University of Wageningen, The Netherlands.

Zonneveld, J.S. (1949). Zand-petrologische onderzoekingen in de terrassen van Zuid-Limburg. Med. Geol. Stichting N.S., 3, 103-123.

\section{Coordonnées des auteurs :}

Étienne JUVIGNÉ

ULiège Département de Géographie Unité de Géographie physique ejuvigne@skynet.be

Geoffrey HOUBRECHTS

ULiège Département de Géographie Unité de Géographie physique - UR Sphères G.Houbrechts@uliege.be 
ANNEXE

\begin{tabular}{|c|c|c|c|c|c|c|c|c|c|c|}
\hline & Lambeaux de terrasse & Dét & Zir & $\mathrm{TiO} 2$ & Tour & Para & Epid & Gre & Amp & $n=$ \\
\hline & Dinant-Huy & & & & & & & & & \\
\hline 1 & Hun (151 m; d64) & LB & 40,9 & 22,7 & 22,7 & 9,1 & - & 4,5 & 0,0 & 44 \\
\hline 2 & Hun $(127 m ; d 40)$ & LB & 28,9 & 22,2 & 20,0 & 4,4 & 2,2 & 4,4 & 17,8 & 45 \\
\hline 3 & Profondville (122m; d37) & LB & 28,7 & 22,3 & 25,7 & 13,5 & 1,4 & 4,1 & 4,4 & 296 \\
\hline 4 & Tailfer (90m; d5) & LB & 23,2 & 17,9 & 17,9 & 7,1 & 1,8 & 30,4 & 1,8 & 56 \\
\hline 5 & Wépion-Onx (200m; d116) & LB & 8,0 & 14,6 & 68,3 & 9,0 & - & - & 0,0 & 199 \\
\hline 6 & Wépion (107 m; d23) & LB & 24,7 & 20,1 & 30,5 & 8,6 & 2,9 & 3,4 & 9,8 & 174 \\
\hline 7 & Jambes-cimetière (b115;s120;d35) & EJ & 30,5 & 9,0 & 35,3 & 10,2 & 4,2 & 0,6 & 10,2 & 167 \\
\hline 8 & Chemin d'Erpent (b110;s115;d31) & EJ & 30,0 & 6,3 & 43,2 & 4,7 & 3,7 & 2,1 & 10,0 & 190 \\
\hline 9 & Lives-sur-Meuse (b135;s140;d56) & EJ & 9,6 & 16,9 & 55,9 & 9,6 & 5,6 & 2,3 & 0,0 & 177 \\
\hline 10 & Namèche (171m) & EJ & 25,1 & 7,7 & 58,5 & 6,7 & 0,5 & 1,5 & 0,0 & 195 \\
\hline 11 & Brichebo (187 m) & EJ & 45,0 & 7,0 & 35,1 & 11,1 & 0,4 & 1,5 & 0,0 & 271 \\
\hline 12 & Petit-Waret (b213;s216) & EJ & 49,7 & 10,7 & 21,4 & 14,4 & 3,2 & 0,5 & 0,0 & 187 \\
\hline 13 & Troka $(206 \mathrm{~m})$; Petit-Waret & LB & 14,0 & 20,1 & 33,0 & 24,6 & 8,4 & - & 0,0 & 179 \\
\hline 14 & Petit-Waret est (b213;s216) & EJ & 28,1 & 4,1 & 46,3 & 14,9 & 5,0 & 0,8 & 0,8 & 121 \\
\hline 15 & Sclayn-Rouvroy (b?;s175) & EJ & 13,2 & 4,7 & 70,8 & 10,4 & 0,3 & - & 0,6 & 318 \\
\hline 16 & Landenne $(183 \mathrm{~m})$ & EJ & 32,2 & 6,7 & 47,6 & 13,0 & 0,5 & - & 0,0 & 208 \\
\hline 17 & Seilles (b165-169) & LB & 14,3 & 27,5 & 31,3 & 26,9 & - & - & 0,0 & 182 \\
\hline 18 & Seilles (b148;s155) & LB & 14,3 & 19,3 & 39,5 & 24,4 & - & 1,7 & 0,8 & 119 \\
\hline 19 & Seilles (b113;s117) & LB & 11,2 & 15,5 & 33,5 & 22,3 & 2,9 & 6,3 & 8,3 & 206 \\
\hline 20 & Surlemez (b213;s216) & EJ & 30,2 & 12,3 & 36,8 & 18,9 & 0,9 & 0,9 & 0,0 & 106 \\
\hline \multirow[t]{2}{*}{21} & Paradis (b175;s179;d103) & EJ & 31,2 & 5,6 & 40,8 & 20,9 & 1,0 & 0,4 & 0,0 & 1276 \\
\hline & Basse-Meuse (flanc droit) & & & & & & & & & \\
\hline 22 & Bois de Breux-1 (b186;s194;d126) & EJ & 57,3 & 9,8 & 12,9 & 20,0 & - & - & 0,0 & 255 \\
\hline 23 & Bois de Breux-2 (b184;s186;d124) & EJ & 40,7 & 6,9 & 30,2 & 20,6 & 1,6 & - & 0,0 & 189 \\
\hline 24 & Bois de Breux-3 (b178;s183;d118) & EJ & 20,1 & 8,0 & 51,4 & 20,4 & - & - & 0,0 & 323 \\
\hline 25 & Jupille (b60;s72;d0) & EJ & 57,6 & 3,9 & 12,6 & 2,6 & 2,2 & 9,1 & 12,0 & 460 \\
\hline 26 & Rabosée (b160;s165;d101) & EJ & 35,5 & 4,3 & 44,3 & 13,9 & 1,7 & 0,3 & 0,0 & 352 \\
\hline 27 & Hognée (b145;s154;d86) & EJ & 49,1 & 8,3 & 24,0 & 13,0 & 5,6 & - & 0,0 & 338 \\
\hline 28 & Barchon (b172,s182;d113) & EJ & 27,7 & 7,6 & 43,6 & 20,5 & 0,6 & - & 0,0 & 512 \\
\hline 29 & La Tombe/Dahlem (b129;s139;d74) & LB & 9,5 & 10,1 & 17,6 & 6,4 & 1,4 & 3,0 & 52,0 & 296 \\
\hline \multirow[t]{2}{*}{30} & Génistreux (b108;s112;d52) & LB & 25,4 & 12,7 & 31,9 & 6,1 & 3,8 & 8,0 & 12,2 & 213 \\
\hline & Basse-Meuse (flanc gauche) & & & & & & & & & \\
\hline 31 & Herstal (b63;s67;d3) & EJ & 41,6 & 5,1 & 12,7 & 10,9 & 2,4 & 10,7 & 16,7 & 534 \\
\hline 32 & Pontisse (b118;s124;d60) & LB & 14,0 & 13,3 & 20,8 & 6,5 & 2,8 & 6,5 & 36,1 & 399 \\
\hline 33 & Haccourt (b60;s72;d3) & EJ & 12,8 & 1,9 & 28,2 & 3,2 & 5,1 & 13,5 & 35,3 & 156 \\
\hline 34 & Plaine alluviale à Visé (b46;556) & EJ & 47,4 & 5,8 & 16,8 & 2,2 & 3,6 & 23,4 & 0,7 & 137 \\
\hline 35 & Heure-le-Romain (b112;s120;d56) & EJ & 18,7 & 2,9 & 19,0 & 4,1 & 3,4 & 6,5 & 45,4 & 443 \\
\hline 36 & Lixhe (b134;s140;d80) & EJ & 34,1 & 4,2 & 41,4 & 12,4 & 2,4 & 1,0 & 4,5 & 1205 \\
\hline 37 & Lanaye (b110;s118;d59) & EJ & 41,6 & 6,6 & 26,1 & 5,8 & 2,2 & 3,5 & 14,2 & 226 \\
\hline \multirow[t]{2}{*}{38} & Romont-3 (b92;s98;d40) & EJ & 25,2 & 5,6 & 45,4 & 6,3 & 5,0 & 0,7 & 11,9 & 302 \\
\hline & Limbourg (flanc droit) & & & & & & & & & \\
\hline 39 & Beitel (s190-200) & LB & 17,5 & 19,0 & 38,0 & 24,8 & 0,7 & - & 0,0 & 137 \\
\hline 40 & Kosberg (b188-189) & LB & 26,8 & 12,6 & 38,4 & 21,5 & 0,7 & - & 0,0 & 549 \\
\hline 41 & Valkenburg (b102;s112) & LB & 10,3 & 7,1 & 16,7 & 6,3 & 1,6 & 3,2 & 54,8 & 126 \\
\hline \multirow[t]{2}{*}{42} & Elsloo (b47;s51) & LB & 5,4 & 7,4 & 33,9 & 7,8 & 5,8 & 5,4 & 34,2 & 257 \\
\hline & Limbourg (flanc gauche) & & & & & & & & & \\
\hline 43 & Pt Campine Sibelco b80;s90) & LB & 11,1 & 11,1 & 39,5 & 7,6 & 5,8 & 3,7 & 21,1 & 602 \\
\hline 44 & P Campine As (b75;s90) & LB & 16,0 & 13,8 & 39,4 & 6,0 & 4,3 & 1,2 & 19,3 & 419 \\
\hline 45 & Pt Campine Lanklaar (b77;s92) & LB & 4,6 & 12,1 & 53,1 & 5,9 & 4,2 & 2,3 & 17,9 & 307 \\
\hline 46 & Lanaken (b61;s65) & LB & 16,2 & 7,8 & 19,1 & 4,0 & 2,9 & 2,9 & 47,1 & 346 \\
\hline 47 & Lanaken (Veldwezelt, site archéo.) & EJ & 17,8 & 4,4 & 31,9 & 9,6 & 10,2 & 7,0 & 19,0 & 342 \\
\hline 48 & Caberg-Pietersem, Riss I (b48;s53) & LB & 8,3 & 17,4 & 18,1 & 7,3 & 7,3 & 2,5 & 39,0 & 436 \\
\hline 49 & Mérode Riss II (b42;s50) & LB & 21,1 & 15,9 & 27,6 & 7,7 & 7,7 & 10,6 & 9,3 & 246 \\
\hline 50 & Eisden-Lanklaar (b42;s52) & LB & 17,4 & 36,2 & 22,9 & 5,0 & 4,6 & 8,1 & 5,7 & 541 \\
\hline 51 & Maasmechelen (s42) & LB & 31,7 & 19,8 & 20,8 & 6,9 & - & 20,8 & 0,0 & 101 \\
\hline
\end{tabular}

Tableau 1. Associations des minéraux denses transparents $(75-425 \mu \mathrm{m})$ de terrasses de la Meuse depuis Dinant jusqu'au Limbourg. 
